\title{
WILEY-VCH
}

\section{Design Criteria for Horseshoe and Spiral-based Interconnects for Highly Stretchable Electronic Devices}

\author{
Dr. Nadeem Qaiser ${ }^{1}$, Asrar Nabil Damdam ${ }^{1}$, Dr. Sherjeel Munsif Khan ${ }^{1}$, \\ Saleh Bunaiyan ${ }^{1}$, Prof. Muhammad Mustafa Hussain ${ }^{1,2 *}$ \\ ${ }^{1}$ mmh Labs, Electrical Engineering, Computer Electrical and Mathematical Science and \\ Engineering Division, King Abdullah University of Science and Technology (KAUST), \\ Thuwal 23955, Saudi Arabia, Tel.: +966-544-700-072 \\ ${ }^{2}$ EECS, University of California, Berkeley, CA 94720, USA.
}

*Corresponding author; E-mail: mmhussain@berkeley.edu /

muhammadmustafa.hussain@kaust.edu.sa

Keywords: stretchable electronics, spiral, horseshoe, FEM, materials, fabrication

\section{ABSTRACT}

Stretchable electronics can be used for numerous advanced applications such as soft and wearable actuators, sensors, bio-implantable devices, and surgical tools because of their ability to conform to curvilinear surfaces, including human skin. The efficacy of these devices depends on the development of stretchable geometries such as interconnection-based configurations and the associated mechanics that help achieve optimum configurations. This work presents the essential mechanics of silicon ( $\mathrm{Si}$ ) island-interconnection structures, which include horseshoe and spiral interconnections, without reducing the areal efficiency. In particular, this study demonstrates the range of the geometrical parameters where they have a high stretchability and cyclic life. The numerical results predict the areas that are prone to breaking followed by experimental validation. The merit for these configurations is achieved by mapping the fracturefree zones for in-plane and out-of-plane stretching with essential implications in stretchable and wearable system design. Furthermore, this work demonstrates the mechanical response for a range of materials (i.e., copper, gold, aluminum, silver, and graphene) that experience the 


\section{WILEY-VCH}

plastic deformations in contrast to conventionally used Si-based devices that represent the extended usage for advanced stretchable electronic devices. The detailed mechanics of these configurations provides comprehensive guidelines to manufacture wearable and stretchable electronic devices.

\section{Introduction}

Stretchable electronics is a newly established technology that provides conformable, flexible, and bendable electronic components. Recent studies have tried to render these electronic components for bending as well as in-plane or axial stretching. These stretchable and flexible devices can potentially be used for several state-of-the-art applications that includes: wearable electronic devices or electronic skin, ${ }^{[1-4]}$ reconfigurable and paper-like displays, ${ }^{[5-9]}$ health-monitoring sensors/actuators, ${ }^{[10,11]}$ photodiodes, ${ }^{[12]}$ electronic eyes, ${ }^{[13]}$ solar cells, ${ }^{[14-16]}$ stretchable integrated circuits/circuit boards, antennas, ${ }^{[17-20]}$ energy harvesters, ${ }^{[21]}$ and soft robotics. ${ }^{[22-24]}$ Clinical procedures that are based on the feedback from a health monitoring system need the rigid components to have intimate integration with human tissues. ${ }^{[25-27]}$ As a result, one of the crucial requirements includes the capability to sustain large deformations while upholding higher stretchability. There are several strategies to attain stretchability in noncompliant inorganic materials, i.e., embedding the rigid components into pre-stretched elastomeric substrates such as polydimethylsiloxane (PDMS) and Ecoflex. These inorganic components not only consist of complementary metal-oxide-semiconductor (CMOS) compatible silicon ( $\mathrm{Si}$ ), but they also consist of metals like copper, gold, and aluminum that are equally beneficial for different applications, e.g., a stretchable antenna. A wave-shaped $\mathrm{Si}$ accommodated 15-20\% strain when embedded into PDMS, or it withstood the strain by $100 \%$ when it was bonded selectively with an augmented design. ${ }^{[28,29]}$ Likewise, a metal-based thin film (e.g., gold) was placed into the PDMS and was stretched by $100 \%$ with an insignificant change in the resistance. ${ }^{[30]}$ 


\section{WILEY-VCH}

Alternatively, to attain stretchability, the strategies consist of reducing the size of bulk Si to the submicron level (ultra-thin $\mathrm{Si}$ ) and controlling the neutral plane. ${ }^{[31]}$ These techniques enhanced the mechanical response of stretchable actuators and sensors by reducing the bending strain ${ }^{[32]}$ However, these techniques are slightly tricky because of the limitation of thickness reduction. Another prevailing technique to resolve the large deformations involves using certain thin geometrically reversible layouts. These reversible configurations with optimized parameters provide geometrically based stretchability, which enables stretchable sensors, actuators, circuits, and transistors. For instance, an island-interconnection configuration is used, wherein all of the active electronic components are placed on the island. Meanwhile, the interconnection acts as a buffer to accommodate the large strains. The design layouts for the interconnections, which include spiral, meander, serpentine, fractal, and horseshoe, can accommodate large deformations. ${ }^{[33-36]}$

It is well known that the metal interconnections (i.e., copper and gold) that are used in stretchable circuits provide electronic conduction in the components. However, we emphasize a situation in which Si-based active devices that are placed on top of Si-based islands are integrated onto the same bare-Si wafer that requires the CMOS-based fabrication flow. Now, the question that arises is how to achieve stretchability while connecting numerous islands. One solution is to provide the Si bridge (interconnection) between consecutive islands. Therefore, we demonstrate Si-based interconnections first rather than use common metal ones, which are useful for providing the required stretchability. We chose the spiral and horseshoe geometries over the other geometries because they have distinct features in comparison to their counterparts. For instance, spiral geometries can use multi-turn arms that wrap around the inner circle; thus, they provide stretchability up to the manifold. Likewise, the horseshoe design offers high mechanical reliability. Most researchers adopt these configurations and demand the optimization of the spiral and horseshoe configurations. Recently, spiral and horseshoe-shaped interconnections have served as exemplary configurations for a variety of applications such as 


\section{WILEY-VCH}

sensing, $360^{\circ}$ imaging, and thermotherapy, because of their higher stretchability, reversibility, engineering control, and compatible fabrication. ${ }^{[12,37-39]}$ Moreover, these stretchable interconnections can act as a way to provide stretchability to a semiconductor material (e.g., $\mathrm{Si})$, but they might act as an electrical-interconnection when a metal can be deposited at the targeted areas of the device. The primary methodology to underpin stretchability requires an understanding of the underlying mechanisms. Although previous studies have reported the applications and associated mechanics for similar configurations, the detailed failure criteria for the island-interconnection structure needs to be addressed. ${ }^{[34,40-43]}$ Moreover, previous studies have mainly discussed the shape of the horseshoe serpentine and its parameters, i.e., the effect of the number of serpentines, their radius, and angle. However, changing these parameters could decrease the areal efficiency of the structures. Most importantly, these studies covered the mechanical response of only a few materials, (i.e., Si and copper), which may not address the broad areas of the applications. Therefore, there is a dire need to find the optimum interconnection-based stretchable configurations. This especially applies to constituent materials that have a high mechanical integrity for advanced applications.

Here, we establish how the basic units of the islands with horseshoe and spiral interconnections respond during stretching until they experience a mechanical fracture. This study presents an in-depth analysis, and this is achieved by changing the thickness and width parameters while maintaining the same areal efficiency. We fabricated the horseshoe and spiral configurations to test their functionalities and to reveal their mechanics. The detailed mechanics of these configurations can provide comprehensive guidelines to manufacture wearable and stretchable electronic devices from the previous stages. In particular, this study reveals the mechanical response of these configurations with a wide range of materials such as $\mathrm{Si}$, aluminum, silver, copper, gold, and graphene, which are crucial for manufacturing pertinent stretchable devices. Our numerical and experimental results show that the mechanical integrity is mainly affected by the thickness of the island-interconnection configuration. By contrast, the 


\section{WILEY-VCH}

width plays an insignificant role. The calculations show that these structures also perform well during out-of-plane stretching. Furthermore, compliant metals undergo plastic deformation, which has extended stretchability. The results also explain why graphene has a higher stretchability for horseshoe and spiral interconnections than that of other metals, whereas silver has stress fluctuations, which results in a lower mechanical integrity. For a variety of wearable, biomedical, display, and other stretchable electronic devices, this study provides crucial guidelines when choosing a particular size, shape, and material with a high form factor.

\section{Results and Discussion}

\subsection{Mechanics of Horseshoe and Spiral Interconnections}

During the functioning of these interconnections, tensile in-plane stretching induces deformations and stress/strain in the structure. The interconnection also experiences bending at the ends of the arm that connect to the inner circle of the spiral interconnection. ${ }^{[38]}$ In general, the interconnection of a given shape, size, and material will experience the fracture once the induced bending strain exceeds the intrinsic fracture strain of the material. The mechanical integrity of the thin structures increases as the bending strain and energy release rate decrease linearly, whereas the flexural rigidity reduces with the cube's thickness of the interconnection. ${ }^{[4]}$ Thus, deterministically engineered configuration of the interconnections with a variety of materials would define the figure-of-merit by providing the fracture-free zone.

Advanced applications use complex arrays that consist of multiple islandinterconnections. We previously demonstrated that the mechanical response of these interconnection-based complex arrays can be determined by taking a single unit cell. ${ }^{[39]}$ Therefore, to obtain the optimum performance from these devices, it is essential to understand the basic cell of these interconnections. Figure 1 shows the schematics of the basic cell of the island-interconnection configuration, i.e., the horseshoe and spiral structures and their associated parameters. Figure 1a shows a spiral anchored between two hexagonal islands. It is 


\section{WILEY-VCH}

well known that the bending strain $\left(\varepsilon_{b}\right)$ is related to the thickness $(\mathrm{t})$ of the structure and the radius of curvature $(\mathrm{R})$ as

$$
\varepsilon_{b}=\frac{t}{2 R}
$$

Previous studies have used the above relation to design the spiral by taking $R$ as the radius of the inner circle as shown in Figure 1a. ${ }^{[45,46]}$ The interconnection experiences mechanical deformations and stress when it is subjected to stretching. We might compare the induced principal strain with the inherent fracture strain of the material (i.e., $1 \%$ for $\mathrm{Si}$ ) to determine if the interconnection will fail. Likewise, the von Mises stress $(\sigma)$ criterion is used to determine if a given design will fail, i.e., the induced $\sigma$ exceeds the fracture strength of a given material. Furthermore, as semiconductor materials such as Si experience elastic deformations (i.e., $\sigma$ changes linearly with the strain), Hook's law can dictate the failure in terms of the evolved $\sigma$ as expressed by the following relation

$$
\sigma=(E) * \frac{t}{2 R}
$$

where $E$ represents the Young's modulus of Si. Equations (1) and (2) can define the fracture conditions of the structure. For example, the calculations from Equation (2) indicate that the spiral of Si with $t=5 \mu \mathrm{m}, R=250 \mu \mathrm{m}$, and $E=170 \mathrm{GPa}$ should fail at the evolved $\sigma$ of 1.7 GPa. To obtain a high form factor, we modified the size of $t$ rather than the size of $R$. This is because doubling $R$ can make the interconnection too large to obtain a high areal efficiency for the spiral-based stretchable system. Figure $1 \mathrm{~b}$ shows a certain horseshoe structure that is anchored between two square islands. Next, to optimize the horseshoe and spiral, we chose the out-of-plane dimension, that is, the width $(w)$ of the interconnections. The initial length $\left(L_{\mathrm{i}}\right)$ of the interconnection corresponds to the gap between the two islands in the undeformed state. When the islands are subjected to tensile displacement $\left(D_{\mathrm{p}}\right)$, the interconnection arm starts to unwrap. Arc length $\left(L_{\mathrm{a}}\right)$ can be regarded as the length of the interconnection in the fully stretched state, i.e., when it becomes fully rectilinear. During stretching, instantaneous 


\section{WILEY-VCH}

stretchability can be defined as the ratio between the initial gap and the change in the gap between the two islands. Note that that the maximum stretchability or opening of the interconnection is limited by its $L_{\mathrm{a}}$. In other words, the maximum stretchability offered by the design can be defined as

$$
\text { Max.stretchability (\%) by design }=\frac{L_{a}-L_{i}}{L_{a}} \mathrm{X} 100
$$

We define the ratio of $D_{\mathrm{p}}$ and $L_{\mathrm{i}}$ as the applied stretch. In regard to the material's effect, a given interconnection might be stretched beyond the limits of $\mathrm{L}_{\mathrm{a}}$ depending on how much material is intrinsically malleable. However, it is expected that the cross-sectional area of the interconnection will be reduced simultaneously, and then it will permanently deform (i.e., it will not revert to the original shape after releasing the load). As a result, the interconnection will ultimately break, once its thickness dramatically decreases, i.e., the neck is formed. First, we consider Si for both of the interconnections, and this shows their figure-of-merit for in-plane and out-of-plane stretching by revealing the underlying mechanics. The effect of the intrinsic material properties will be discussed in a later section.

\subsection{Failure Criteria for Si-based Interconnections}

We fabricated the island-interconnection structures by using the process that is described in Figure S1 (S refers to the supplementary information henceforth). During fabrication, we deposited amorphous-Si, which is referred to as Si afterward. The experimental setup and initially undeformed state of the fabricated sample are shown in Figure S2a. Figures 2(a-c) show the stretching profiles of the fabricated horseshoe for a constant $t$ and $w$ of 5 and $10 \mu \mathrm{m}$, respectively, during the various stages of stretching at $67 \%, 164 \%$, and $295 \%$, respectively. The results show that the horseshoe readily opens along the axial direction. Next, we compare the numerical calculations of the horseshoe by making the parameters comparable to the fabricated structures. Figures 2(d-f) illustrate the deformation contours by applying the finite element method (FEM) when the interconnection is subjected to $D_{\mathrm{p}}$ for a similar stretch. 


\section{WILEY-VCH}

The stretching profiles that are obtained by the experiments and the FEM match each other, and they validate the numerical calculations. As it is challenging to experimentally measure the real-time $\sigma$ states during stretching, we use the FEM calculations to our advantage to calculate the 3D stress and strain evolution during a similar stretch, as shown in Figures S3(a-c). The inset shows the enlarged images that reveal the $\sigma$ distribution along the boundary of the interconnection. The area of $\sigma$ concentration (red-colored areas) shows a region that is expected to break first.

Further, we continue the stretching of the horseshoe until it experiences cracks (i.e., its induced principal strain exceeds $1 \%$ ). Figure $2 \mathrm{~g}$ shows the optical images of the horseshoe at the onset of the fracture, which has a stretch of $408 \%$. The inset of Figure $2 \mathrm{~g}$ reveals the cracked horseshoe. Figure $2 \mathrm{~h}$ shows the deformations that are calculated by the FEM, and Figures 2(ij) illustrate the $\sigma$ and principal strain contours at the same stretch of $408 \%$, in which the zoomed images are shown in the inset. At the onset of fracture, the maximum magnitude of $\sigma$ reaches $\sim 1.7 \mathrm{GPa}$ at a corresponding strain of $1 \%$. It is worth mentioning that the horseshoe was cracked at the same location as predicted by the FEM calculation, i.e., the area of the $\sigma$ concentration (see Figure S3). In addition, the $1.7 \mathrm{GPa}$ magnitude of $\sigma$ that was attained by the FEM exactly matches the theoretically calculated $\sigma$ that was determined by Equation (2).

Furthermore, we follow the same pattern for the spiral, that is, the fabricated spiral structure with a constant $t$ and $w$ of 5 and $10 \mu \mathrm{m}$, respectively. We compared the experimental and FEM results, and as shown, Figures 3(a-c) demonstrate the experimental stretching profiles of the spiral for the applied stretch of $44 \%, 95 \%$, and $150 \%$, respectively. The undeformed state for the experimental stretch is shown in Figure S2c. Figures 3(d-f) present the numerical results of the deformations. Figures $\mathrm{S} 4(\mathrm{a}-\mathrm{d})$ reveal the contours of $\sigma$ for the corresponding applied stretches of $44 \%, 95 \%, 132 \%$, and $150 \%$, respectively. It is logical to comprehend that due to the bending at the region where the arm connects to the inner circle, the spiral can intercept the fracture conditions, even before it reaches $L_{\mathrm{a}}$. The zoomed images clarify the location of the $\sigma$ 


\section{WILEY-VCH}

concentration, which primarily depends on the topology of the interconnection. Interestingly, by looking at the value of $\sigma$, we can predict that the spiral would break upon further stretching because the induced $\sigma$ (i.e., $1.62 \mathrm{GPa}$ ) is close enough to theoretically estimate the fracture $\sigma$ of 1.7 GPa. As expected, further stretching (154\%) results in the failure of the spiral, as shown in the optical image in Figure 3g. The inset shows a spiral structure just before the fracture. It is interesting to note that it was experimentally broken at the same location (see the supplementary video) that was predicted from the $\sigma$ concentration region that is based on the FEM calculations. Figures 3 (h-j) illustrate the deformation, $\sigma$, and the principal strain contours at the corresponding stretch of $154 \%$, respectively, and the zoomed images are shown in the inset. Because the experimental and FEM results match well, we performed several simulations for a range of $t$ and $w$ for the horseshoe and spiral interconnections and devised a figure-of-merit. We defined the cutoff value of $t$ where the resultant stretchability decreases up to $40-50 \%$ for the respective interconnections.

\subsection{Design Criteria and Figure-of-Merit for Si-based Interconnections}

First, we set the constant $w$ as $10 \mu \mathrm{m}$ and changed the $t$ of the horseshoe and spiral arm as $3 \mu \mathrm{m}, 5 \mu \mathrm{m}, 7 \mu \mathrm{m}$, and $10 \mu \mathrm{m}$, respectively (Figures $4 \mathrm{a}$ and $\mathrm{b}$ represent the definition of $t$ and $w$ of the arm). The parameters and the definition of the normalized length are also shown in the figure. For the horseshoe-arm, we extracted the results during an applied stretch of $125 \%$, $300 \%$, and at the onset of the fracture. We considered the highest magnitude of $\sigma$ in the interconnection that might evolve at any region along the boundary of the arm and recorded it as the maximum von Mises stress $\left(\sigma_{\max }\right)$. For the horseshoe-arm, Figure $4 \mathrm{c}$ shows the evolution of $\sigma_{\max }$ as a function of $t$. The results indicate that $\sigma_{\max }$ significantly increases with an increasing $t$ with an applied stretch of $125 \%$ and $300 \%$. Nevertheless, regardless of the arm $t, \sigma_{\max }$ does not change considerably once the arm intercepts the fracture conditions. Likewise, for the spiral arm, the evolution of $\sigma_{\max }$ during the applied stretch of $20 \%, 50 \%, 75 \%$, and at the onset of fracture is shown in Figure $4 \mathrm{~d}$. The spiral also experiences nearly the same $\sigma_{\max }$ when they attain 


\section{WILEY-VCH}

the fracture $\sigma$, regardless of the value of $t$. However, before that point, $\sigma_{\max }$ changes linearly with the thickness in the spiral.

It is necessary to map $\sigma$ along the arm's boundary because it can predict the region that is prone to breaking. Thus, we defined the normalized length, as shown in Figures 4a and b, along with the definition of the 0 and 1 positions. Next, for each value of $t$, we extracted $\sigma$ along the normalized length of the horseshoe arm, as shown in Figure 4e. It is interesting to show that the arm evolves the nonuniform $\sigma$ along its boundary. Although the value of maximum $\sigma$ is almost comparable for each $t$, the overall magnitude of $\sigma$ is lower for a thin horseshoe than for that of a thicker counterpart. At the maximum stretch, the evolution of the displacement and principal strain (\%) along the normalized length of the horseshoe arm is shown in Figure S5. Figure 4 f exhibits the distribution of $\sigma$ along the normalized length of the spiral arm. The results claim that the area of the arm near the inner circle induces the highest $\sigma$, which indicates the region that is prone to breaking first. Interestingly, the optical image shown in Figure $3 \mathrm{~g}$ verifies that the spiral was broken in the predicted region (Figure S4). Under the fracture conditions, the detailed evolution of the displacements, strain along the normalized length, and 3D stress/strain contours for the spiral are shown in Figure S6. The results claim that, under the fracture conditions, the magnitude of the stress is almost identical for each value of $t$; however, a thicker spiral $(t=10 \mu \mathrm{m})$ unwraps to a lesser extent, that is, representing low stretchability in comparison to the thin spiral $(t=3 \mu \mathrm{m})$.

To investigate this further, we considered different $t$ and $w / t$ ratios (i.e., 2 and 10) and demonstrated that $\sigma_{\max }$ is a function of stretching in Figure $4 \mathrm{~g}$. Our results reveal that for the case of $t=3 \mu \mathrm{m}, \sigma_{\max }$ is lower with an increasing amount of stretching until the horseshoe opens more than $90 \%$ of its $L_{\mathrm{a}}$. Upon further stretching, $\sigma_{\max }$ evolves exponentially, which leads to a fracture. In contrast, for $t=10 \mu \mathrm{m}, \sigma_{\max }$ increases almost linearly with an increasing amount of stretching. Another useful piece of information is that for $t=3 \mu \mathrm{m}$, the horseshoe is expected to have a high mechanical integrity if it is stretched within $90 \%$ of its maximum stretchability 


\section{WILEY-VCH}

since it experiences a low $\sigma_{\max }$ in this range. We considered that the ratio of $w / t$ is 10 for the spiral because the results for the horseshoe show that the results are nearly identical for w/t of 2 and 10. The evolution of $\sigma_{\max }$ with respect to the applied stretching for different $t$ and $w / t$ ratios of 10 is shown in Figure $4 \mathrm{~h}$. Interestingly, $\sigma_{\max }$ started to increase for the spiral at the start of the stretching process. However, unlike $t=10 \mu \mathrm{m}, \sigma_{\max }$ remains lower for $t=3 \mu \mathrm{m}$. These results are consistent for both cases, which shows that thin interconnections have a higher mechanical integrity.

Furthermore, for the horseshoe structure, we extended the range of $t$ up to the point where its stretchability goes down to a fair amount (i.e., lower than $40-50 \%$ ), as shown in Figure 5a. For a given range of $t$, the area under the curve outlines the figure-of-merit by showing the fracture-free region of the horseshoe. For $t=3 \mu \mathrm{m}$, the stretchability was $410 \%$, which decreased to $392 \%$ at $t=10 \mu \mathrm{m}$. After that, it adversely reduces to $184 \%$ at $t=25 \mu \mathrm{m}$, which shows that the stretchability is approximately $55 \%$ less than that at $t=3 \mu \mathrm{m}$. The results suggest that the horseshoe up to a $t$ of $10 \mu \mathrm{m}$ provides a high efficacy, as the stretchability does not change much. Therefore, the stretchable devices might utilize the $t$ up to $10 \mu \mathrm{m}$ to achieve highly stretchable designs. It can be challenging to deposit a thicker Si to reveal the effect of $w$ experimentally due to the long fabrication time and the high cost of the plasma-enhanced chemical vapor deposition (PECVD) process. Nevertheless, provided that the results of the FEM are well matched with our experiments, we used the numerical calculations to reveal the effect of $w$, that is, from 10 to $100 \mu \mathrm{m}$ (Figure 5b). Interestingly, the results claim that $w$ has an insignificant effect on the performance of the horseshoe, that is, it has less than a $1 \%$ change in the stretchability.

Next, we calculated the same for the spiral case, and the results are shown in Figures 5c $\mathrm{d}$. The stretchability of the spiral decreased from $159 \%$ at $t=3 \mu \mathrm{m}$ to $88 \%$ at $t=10 \mu \mathrm{m}$. As the out-of-plane deformations are equally important, we performed numerical calculations to map the figure-of-merit during out-of-plane stretching. Figures 5e and f show the out-of-plane 


\section{WILEY-VCH}

stretching results for the horseshoe and spiral, respectively. The results show that the horseshoe and spiral have a comparable performance to in-plane stretching. Among both structures, for the thicker samples of the horseshoe, that is, $t=25 \mu \mathrm{m}$, its stretchability was further reduced to $144 \%$ in comparison to $184 \%$ for in-plane stretching. This could be attributed to the out-ofplane twisting of the interconnection, as shown in Figure S7. However, for the spiral case, the stretchability is identical to in-place stretching because the inner circle provides systematic outof-plane unwrapping of the spiral interconnection. The stress contours for the out-of-plane deformations for both interconnections are shown in Figure S7. Our results claim that the $t$ of the interconnections has a pronounced effect on the performance of the horseshoe and spiral topologies. However, the mechanical performance is nearly unaffected by the variation in $w$ of the interconnections. This is an interesting fact that may help in the design and fabrication of stretchable electronic devices.

\subsection{Reversibility and Design Criteria of Thicker Si-based Interconnections}

As we have outlined the underlying mechanism of the stretching response for a variety of interconnections, it is important to fabricate thicker interconnections to validate the design calculations. The initially undeformed state for the horseshoe with $t=25 \mu \mathrm{m}$ and $w=10 \mu \mathrm{m}$ is shown in Figure S2b. For the thicker samples of the interconnections, we used an in-house built testing setup to eliminate the rotation of the island; we used adhesive tape to hold the islands firmly. Figure 6 a shows that the experimental stretch of $183 \%$ is on the verge of cracking (symbols $S$ and $F$ show the start and finish points of the experimental stretch). By contrast, Figures $6 \mathrm{~b}$ and $\mathrm{c}$ compare the stretching response and the stress evolution by using numerical calculations. We demonstrate the detailed mechanical response during the various stages of stretching (at 95\% and 140\%) until the fractures (at 184\%) are shown in Figures S8 (a-h). The contours show the areas of the horseshoe arm that have $\sigma$ concentrations (which are shown as red circles) that anticipate the mechanical failure in these regions. Interestingly, the experimental failure that is shown in Figure S8g exactly substantiates the prediction. A 


\section{WILEY-VCH}

comparison of the experimental and FEM stretching for both sizes of the horseshoe until breakage is displayed in SMovie 1. Figure S2d shows the undeformed state, and Figures S9 (af) present the experimental stretching and FEM calculations with an applied stretch of 34 and $66 \%$, respectively, for the fabricated spiral with $t=10 \mu \mathrm{m}$. Figures $6(\mathrm{~d}-\mathrm{f})$ compare the experimental stretch and the FEM while on the verge of fracturing (90\%). The results for the spiral affirm that the arm fails near the inner-circle region, as shown in SMovie 2. Although, it is well established that for reversible cycles, the change in the resistance is insignificant for these stretchable interconnections. This determines the number of cycles that are sustained before the fracture, which is crucial for revealing the sustainability of the structures. We tested the horseshoe and spiral structures by using an in-house built setup. Figures $6 \mathrm{~g}$ and $6 \mathrm{~h}$ illustrate the results for both cases, respectively. SMovie3 and SMovie 4 show the last few cycles before the fracture. The results show that the micron-scale interconnections are highly reversible, and they do not break for more than 400 cycles.

\subsection{Material Perspective of the Interconnections}

Currently, advanced stretchable electronic devices that are intended for textiles, communication, displays, and medical fields require non-Si-based interconnections, that is, they are based on cost-effective metals that offer a high efficacy. Although metals, in contrast to brittle $\mathrm{Si}$, are intrinsically compliant/malleable, their stretchability is still limited and demands stretchable topology. For instance, a metal can be shaped into a stretchable configuration (e.g., serpentine or other interconnections) to obtain advanced functionalities and a higher performance in terms of stretchability. Hence, we consider a range of commonly used materials for the horseshoes and spirals and demonstrate their mechanical response. Unconventional materials, such as graphene, have also emerged as promising candidates for stretchable electronics such as radiofrequency electronics due to their high carrier mobility. ${ }^{[47,48]}$ Previous studies explored the functionalities of metal-based stretchable devices; however, the range of the materials was limited. ${ }^{[30,34,49]}$ Therefore, the materials for the FEM calculations 


\section{WILEY-VCH}

consist of copper, gold, aluminum, silver, and graphene. Although the experimental functioning of these interconnections that are based on these materials necessitates future work, provided that our FEM model predicts correct mechanical response, the underlying mechanics of these malleable materials would provide valuable preliminary work in this field.

Because the taken materials are inherently malleable, they would undergo yielding (deviating from Equation 2) and experience elastic-plastic deformations. We adopt the maximum principal strain criterion to identify the regime when the material experiences plasticity. We considered the ideal elastic-plastic relations and neglected work-hardening, which is a commonly used method to solve similar problems. ${ }^{[35,50]}$ To account for the plastic deformations in the FEM, we incorporated other properties of the materials in addition to their Young's modulus $(E)$ and Poisson's ratio $(v)$, as shown in Table S1. Moreover, to streamline the analysis, we applied a constant $t$ and $w$ of $5 \mu \mathrm{m}$ and $25 \mu \mathrm{m}$, respectively. For a single layer of graphene, the thickness is usually in the nanometer range. However, studies have successfully shown that stacking graphene layers can result in thicker graphene. For instance, Chugh et al. periodically arranged the graphene sheets and dielectric spacers, thus making the thickness of the stacked graphene metamaterial in the range of micrometers. ${ }^{[51]}$ In comparison to a closely packed graphite structure (typically a thin-sheet), the stacked graphene with spacers still offers enough spacing between the layers, which retains the properties of the graphene monolayers. Therefore, we assume that the spacers do not alter the intrinsic material properties of the graphene, and we can take the structural properties of the graphene. As the material properties, especially $E$ and the yield strength for each of the materials, are dissimilar, the resultant $\sigma$ is challenging to compare on one scale. Therefore, for a fair comparison, we $\mathrm{c}$ the normalized $\sigma$, that is, the ratio of the evolved $\sigma$ to its yield strength. We stretch the interconnection of a given material until it reaches its fracture strain, that is, the evolved principal strain becomes comparable to the fracture strain. Therefore, the normalized $\sigma$ is expected to exceed the value of 1 in contrast to the yield-stress criterion approach. The fracture 


\section{WILEY-VCH}

strains of copper, gold, aluminum, silver, and graphene were 5\%, 5\%, 2\%, 20\%, and 22\%, respectively. ${ }^{[52-56]}$

Figures $7 \mathrm{a}$ and $\mathrm{c}$ represent the normalized $\sigma$ values of the horseshoe and spiral arm for the chosen materials. The results show that copper, gold, and aluminum exhibit an analogous response; by contrast, silver and graphene show dissimilar responses. For instance, the induced normalized $\sigma$ for the silver horseshoe arm rises rapidly during the early stage of stretching, and then it saturates due to the plasticity. The identified response continues until the respective interconnection reaches $L_{\mathrm{a}}$ or becomes fully rectilinear. Interestingly, at this point, the silverbased horseshoe arm attains a stretchability of $410 \%$, and the normalized $\sigma$ almost remains in the negligible range for graphene. However, the normalized $\sigma$ suddenly rises after the horseshoe arm becomes rectilinear, that is, it reaches $L_{\mathrm{a}}$. It is remarkable to claim that graphene has an extended stretch in comparison to the other materials. Nevertheless, as the interconnection has already achieved maximum opening that is permitted by the geometrical design, the horseshoe is expected to remain thin beyond this regime. Therefore, the graphene horseshoe reaches $490 \%$ stretchability, while the other materials reach a stretchability of $410 \%$. On the downside, a given interconnection, when exceeding $L_{\mathrm{a}}$, attains a permanent deformation that affects the reversibility. To understand the underlying mechanism, we extracted the evolved strains during the stretch, as discussed later in this section.

Likewise, Figure 7c (for the spiral) shows that the silver-based spiral-arm attains the maximum $\sigma$ at the primary stage of stretching, that is, $25 \%$ of the stretch. By contrast, for graphene, the normalized $\sigma$ remains almost negligible until it is $180 \%$ of the applied stretch. Beyond that, $\sigma$ starts to elevate quickly. The lower $\sigma$ in the spiral arm results in an enhanced stretchability of $214 \%$ for the graphene-based spiral. The chosen materials, including graphene, could not exceed $L_{\mathrm{a}}$ for the spiral, thus proving its lower stretchability. The reason for this could be attributed to the stress concentration in the region where the arm connects to the inner circle of the spiral (i.e., the design is poor in comparison to the horseshoe). 


\section{WILEY-VCH}

We have discussed the strain states to enhance the understanding of the mechanical responses. It is well known that there are typically two types of strains, that is, the principal strain and the plastic strains. The total principal strain contains the accumulative strain of the elastic and plastic strains; however, the plastic strain starts to evolve only once $\sigma$ of the respective material exceeds its yield strength. ${ }^{[57]}$ Figures $7 \mathrm{~b}$ and $\mathrm{d}$ reveal the distribution of the total principal and plastic strains while stretching the horseshoe and spiral, respectively. Figure $7 \mathrm{~b}$ shows that for copper, gold, and aluminum, the horseshoe does not experience any plastic deformation until the applied stretch reaches $300 \%$. We can describe the unusual response of silver and graphene in terms of the evolution of the plasticity. For instance, when the applied stretch is $100 \%$ of the silver-based horseshoe arm, $\sigma$ reaches the yield strength and it starts to undergo plastic deformation. For the graphene-based horseshoe, its arm experiences elastic deformations until the stretch reaches $470 \%$. After that, they experience plastic deformations or nonreversible deformations. Figure $7 d$ reveals the total principal and plastic strains for the spiral. The mechanical response of the spiral-arm with different materials is comparable to the horseshoe-arm, that is, the plastic strain and the normalized $\sigma$ along the normalized length of the arm evolve for each material. It is noteworthy that for a stretch of $215 \%$, the spiral opens up to a permissible stretch by design, that is, $L_{\mathrm{a}}$. The results show that because of the favorable intrinsic material properties of graphene, it exceeds $L_{\mathrm{a}}$; by contrast, other materials fail even before the spiral reaches the limit of $L_{\mathrm{a}}$.

The normalized $\sigma$ of the silver horseshoe arm along the boundary of the normalized length of the arm would be logical to demonstrate. Figure S10a demonstrates that at the onset of the fracture, the normalized $\sigma$ is along the normalized length of the horseshoe-arm. The results show that silver has an unstable or fluctuated $\sigma$ evolution; thus, making it incompatible for stretching applications. In other words, the cyclic life that depends on the fatigue is expected to be lower for the silver-based horseshoe. Likewise, Figure S10b shows the normalized $\sigma$ along the boundary of the spiral. The silver-based spiral arm has a high degree of $\sigma$ severity; the reason 


\section{WILEY-VCH}

for this could be attributed to the bending at the end of the arm that connects to the inner circle. By contrast, the horseshoe experiences a lower $\sigma$ severity due to its favorable topology. The results show that silver is unstable or has $\sigma$ fluctuations, that is, a tooth-saw evolution. This makes it the least favorable choice as a material for the stated stretchable devices. Figures 7e and $f$ summarize the maximum stretchability of the horseshoe and the spiral, respectively, for each of the materials. The results show an enhanced stretchability due to the malleability of the respective material. To provide detailed guidelines for the horseshoe and spiral-based stretchable devices, we have summarized our findings in Table 1, and the parameters are constant. Regardless of the nature of the application, the listed table would be considerably advantageous for the preliminary manufacturing stages of stretchable sensors, actuators, biomedical, communication, and other electronic devices.

\section{Conclusions}

We revealed the underlying mechanics of the horseshoe and spiral-island configurations for stretchable and wearable electronic devices. This was achieved by applying basic mechanics and obtaining numerical and experimental results. We inferred the fracture conditions numerically for a range of horseshoe and spiral arm parameters. We then experimentally verified the regions along the boundary of the interconnection arms that previously experienced fractures. We applied a range of thicknesses and widths for both topologies and revealed their evolved deformation, stress, and strain. For Si-based horseshoes and spirals with thicknesses and widths of $5 \mu \mathrm{m}$ and $10 \mu \mathrm{m}$, respectively, our results demonstrated that their maximum stretchability is $408 \%$ and $154 \%$, respectively. The numerical and experimental results estimated that stretchability decreases with an increasing thickness; thus, there is a reduction in its mechanical integrity. The results suggest that the horseshoe and spiral have a thickness up to $10 \mu \mathrm{m}$ and $5 \mu \mathrm{m}$, respectively. As a result, the horseshoe and spiral provide a high efficacy, which decreases to $50 \%$. Therefore, stretchable devices might be used in this range to obtain highly stretchable designs. We compared the performance of the horseshoe and spiral 


\section{WILEY-VCH}

interconnections when stretching them out-of-plane. We also revealed the reversibility of the interconnections and discovered no fractures for more than 400 cycles, which demonstrates high mechanical integrity of the micron-level samples. We established a detailed FEM analysis for intrinsically malleable materials such as copper, gold, silver, aluminum, and graphene with a constant size to reveal the underlying mechanisms of their stretching. Our results indicate that compliant metals undergo plastic deformation because of their lower yield strength. Among the chosen materials, silver attained a lower stretchability ( $411 \%$ for the horseshoe and $178 \%$ for the spiral) due to $\sigma$ instabilities during the early stage of stretching. By contrast, graphene achieved a higher stretchability (490\% and $214 \%$ for the horseshoe and spiral) compared to the other metals because of its favorable mechanical properties. Our extensive set of results would be useful for determining the fracture conditions for a variety of commonly used materials in addition to Si-based stretchable interconnections for highly stretchable electronic devices, which includes sensors and actuators.

\section{Materials and Methods}

\subsection{Fabrication of Stretchable Horseshoe and Spiral}

We fabricated the interconnection-island structures for the horseshoe and spiral interconnections according to the geometrical shape that is shown in Figure 1. Initially, we used the virtual silicon on insulator (SOI) wafer, as shown in Figure S1. Fabricating the virtual SOI makes the process inexpensive. To achieve this, a layer of oxide $\left(\mathrm{SiO}_{2}\right)$ with a thickness of $10 \mu \mathrm{m}$ was deposited on a p-type Si wafer by a PECVD (Oxford Instruments PlasmaLab Deposition) process. The amorphous silicon $(a-\mathrm{Si})$ was then deposited by using the same process, which completed the fabrication of the virtual SOI wafer. Afterward, we sputtered (Equipment Support Company) aluminum (Al), which was used as a hard mask for the photolithography process. The geometrical shapes of the horseshoe and spiral with respective thicknesses $(t)$ were patterned with the photolithography process (EVG 620S). Al from the wafer was etched by using a reactive ion etching (RIE) process. To obtain the 


\section{WILEY-VCH}

desired width of the samples, we used the deep RIE process (Oxford Instruments PlasmaLab 100). The remaining photoresist (PR) was cleaned by immersing the wafer in acetone. The remaining $\mathrm{Al}$ on the top of the design was etched with a wet $\mathrm{Al}$ etchant for $5 \mathrm{~min}$. The final release of the interconnection-island structures was attained by wet etching with $48 \%$ hydrofluoric acid (HF) (Sigma-Aldrich). Wet etching was performed for $1 \mathrm{~h}$ at room temperature, which allowed isotropic etching of the buried oxide layer; thus, releasing the stretchable network.

\subsection{Numerical Analysis of the Horseshoe and Spiral}

We calculated the mechanical response of the island-interconnection structures by using 3D FEM. Geometrical shapes with a variety of thicknesses were designed in a commercially available computer aided design (CAD) program called SOLIDWORKS ${ }^{\mathrm{TM}}$. These designs were assigned the required width of $10 \mu \mathrm{m}$ along with other ranges when analyzing the width effect. In addition, others were obtained by performing the extrusion function in SOLIDWORKS ${ }^{\text {TM }}$. These geometries were then imported to a commercially available FEM program called ABAQUS ${ }^{\mathrm{TM}}$, which can numerically calculate the underlying mechanics of the samples. The tensile stretch $\left(D_{\mathrm{p}}\right)$ was prescribed at both islands $\left(I_{1}\right.$ and $\left.I_{2}\right)$ from the undeformed state to the fracture condition, that is, the principal strain of the respective material exceeded its intrinsic fracture strain. For out-of-plane stretching, we fixed one island, while prescribing the $D_{\mathrm{p}}$ along the $\mathrm{x}$ and $\mathrm{y}$ axes simultaneously, to provide the required out-of-plane stretching. This analysis predicted the stretching profiles, and it induced the deformation, stress, and strain contours for Si and other materials, which included copper, silver, gold, aluminum, and graphene. The material properties are listed in Table S1. Because the interconnections are expected to experience large deformations, we used "NLgeom on" in ABAQUSTM to address the geometrical nonlinearities. We ensured that the solution achieved convergence by applying a refined mesh, that is, the element size was smaller than the minimum thickness of the arm or the interconnection. 


\section{WILEY-VCH}

\subsection{Mechanical Stretching}

The fabricated samples were experimentally tested to predict their mechanical performance, such as stretching profiles and stretchability. We used two testing methods to prescribe the tensile stretch on the islands of the structures. First, we used a probe station with a high magnification lens to capture the images and probes that were placed on the islands. The tensile stretch was obtained by moving the probes axially outward. This technique is better for thin and delicate samples because the thin samples are radially easy to handle under the mere probe contact. However, for relatively thicker samples, we used an tensile setup built in-house to minimize the rotation of the islands under their own weight. This setup simply consisted of a stage that moved axially while the island on the other side remained fixed with scotch tape. We used the measuring-scale at this stage to ensure the extent of opening of the samples that we used to calculate the total stretchability.

\subsection{Cyclic Testing}

The fabricated configurations were tested for reversibility or cyclability by using an inhouse testing setup. We placed the samples such that they followed the same scheme as the stretching profiles and fixed the islands with scotch tape. The samples were carefully transferred to eliminate out-of-plane bending or deformation. Then, we reversibly stretched and released the samples for several hundred cycles to determine their cyclic life. Because we used a manually operated setup where it is not possible to conduct the reversible stretched/unstretched cycles for several hours, we deliberately broke the samples after they exceeded 400 cycles to stop the cyclic test.

\section{Supporting Information}

Supporting information is available from the Wiley Online Library or from the author.

\section{Acknowledgment}




\section{WILEY-VCH}

This publication is based upon the work that is supported by the King Abdullah University of Science and Technology (KAUST) Office of Sponsored Research (OSR) under Award No. REP/1/2707-01-01 and REP/1/2880-01-01.

\section{Author Contribution}

M. M. H. conceived the idea and directed the project; N. Q. designed the project and conducted the experiments and simulations; A. N. D. assisted in the fabrication; and S. M. K. and S. B. helped manufacture and test the structures.

\section{Competing financial/non-financial interests}

The authors declare that they have no competing financial/non-financial interests. 


\section{WILEY-VCH}

\section{References}

[1] T. Someya, T. Sekitani, S. Iba, Y. Kato, H. Kawaguchi, T. Sakurai, Proc. Natl. Acad. Sci. U. S. A. 2004, 101, 9966.

[2] D.-H. Kim, N. Lu, R. Ma, Y.-S. Kim, R.-H. Kim, S. Wang, J. Wu, S. M. Won, H. Tao, A. Islam, K. J. Yu, T. Kim, R. Chowdhury, M. Ying, L. Xu, M. Li, H.-J. Chung, H. Keum, M. McCormick, P. Liu, Y.-W. Zhang, F. G. Omenetto, Y. Huang, T. Coleman, J. A. Rogers, Science (80-. ). 2011, 333.

[3] K. I. Jang, S. Y. Han, S. Xu, K. E. Mathewson, Y. Zhang, J. W. Jeong, G. T. Kim, R. C. Webb, J. W. Lee, T. J. Dawidczyk, R. H. Kim, Y. M. Song, W. H. Yeo, S. Kim, H. Cheng, S. Il Rhee, J. Chung, B. Kim, H. U. Chung, D. Lee, Y. Yang, M. Cho, J. G. Gaspar, R. Carbonari, M. Fabiani, G. Gratton, Y. Huang, J. A. Rogers, Nat. Commun. 2014, 5 .

[4] T. Yokota, P. Zalar, M. Kaltenbrunner, H. Jinno, N. Matsuhisa, H. Kitanosako, Y. Tachibana, W. Yukita, M. Koizumi, T. Someya, Sci. Adv. 2016, 2.

[5] T. Sekitani, H. Nakajima, H. Maeda, T. Fukushima, T. Aida, K. Hata, T. Someya, Nat. Mater. 2009, 8, 494.

[6] J. Chen, C. T. Liu, IEEE Access 2013, 1, 150.

[7] S. Kim, H. J. Kwon, S. Lee, H. Shim, Y. Chun, W. Choi, J. Kwack, D. Han, M. Song, S. Kim, S. Mohammadi, I. Kee, S. Y. Lee, Adv. Mater. 2011, 23, 3511.

[8] J. A. Rogers, Z. Bao, K. Baldwin, A. Dodabalapur, B. Crone, V. R. Raju, V. Kuck, H. Katz, K. Amundson, J. Ewing, P. Drzaic, Proc. Natl. Acad. Sci. U. S. A. 2001, 98, 4835.

[9] N. Qaiser, S. M. Khan, K. Chow, M. D. Cordero, I. Wicaksono, M. M. Hussain, Adv. Mater. Technol. 2018, 3, 1800344.

[10] S. Imani, A. J. Bandodkar, A. M. V. Mohan, R. Kumar, S. Yu, J. Wang, P. P. Mercier, 


\section{WILEY-VCH}

Nat. Commun. 2016, 7, 11650.

[11] J. Viventi, D.-H. Kim, L. Vigeland, E. S. Frechette, J. A. Blanco, Y.-S. Kim, A. E. Avrin, V. R. Tiruvadi, S.-W. Hwang, A. C. Vanleer, D. F. Wulsin, K. Davis, C. E. Gelber, L. Palmer, J. Van der Spiegel, J. Wu, J. Xiao, Y. Huang, D. Contreras, J. A. Rogers, B. Litt, Nat. Neurosci. 2011, 14, 1599.

[12] G. A. Torres Sevilla, N. Qaiser, M. D. Cordero, S. F. Shaikh, M. M. Hussain, Appl. Phys. Lett. 2018, 113, 134101.

[13] H. C. Ko, M. P. Stoykovich, J. Song, V. Malyarchuk, W. M. Choi, C.-J. Yu, J. B. Geddes III, J. Xiao, S. Wang, Y. Huang, J. A. Rogers, Nature 2008, 454, 748.

[14] C. J. Brabec, N. S. Sariciftci, J. C. Hummelen, Adv. Funct. Mater. 2001, 11, 15.

[15] R. R. Bahabry, A. T. Kutbee, S. M. Khan, A. C. Sepulveda, I. Wicaksono, M. Nour, N. Wehbe, A. S. Almislem, M. T. Ghoneim, G. A. Torres Sevilla, A. Syed, S. F. Shaikh, M. M. Hussain, Adv. Energy Mater. 2018, 8.

[16] N. El-Atab, N. Qaiser, R. Bahabry, M. M. Hussain, Adv. Energy Mater. 2019, 1902883.

[17] J. H. So, J. Thelen, A. Qusba, G. J. Hayes, G. Lazzi, M. D. Dickey, Adv. Funct. Mater. 2009, 19, 3632.

[18] S. Cheng, Z. Wu, P. Hallbjörner, K. Hjort, A. Rydberg, IEEE Trans. Antennas Propag. 2009, 57, 3765 .

[19] M. Kaltenbrunner, G. Kettlgruber, C. Siket, R. Schwödiauer, S. Bauer, Adv. Mater. 2010, 22, 2065.

[20] T. Sekitani, T. Someya, In Conference Proceedings - Lasers and Electro-Optics Society Annual Meeting-LEOS; 2008; pp. 198-199.

[21] R. D. Kornbluh, R. Pelrine, H. Prahlad, A. Wong-Foy, B. McCoy, S. Kim, J. Eckerle, T. Low, MRS Bull. 2012, 37, 246.

[22] Z. Chen, T. I. Um, H. Bart-Smith, Int. J. Smart Nano Mater. 2012, 3, 296. 


\section{WILEY-VCH}

[23] M. Sfakiotakis, D. M. Lane, J. B. C. Davies, IEEE J. Ocean. Eng. 1999, 24, 237.

[24] M. Yamakita, N. Kamamichi, T. Kozuki, K. Asaka, Zhi-Wei Luo, In 2005 IEEE/RSJ International Conference on Intelligent Robots and Systems; IEEE, 2005; pp. 20352040.

[25] K. Zhang, Y. H. Jung, S. Mikael, J.-H. Seo, M. Kim, H. Mi, H. Zhou, Z. Xia, W. Zhou, S. Gong, Z. Ma, Nat. Commun. 2017, 8, 1782.

[26] W. Gao, S. Emaminejad, H. Y. Y. Nyein, S. Challa, K. Chen, A. Peck, H. M. Fahad, H. Ota, H. Shiraki, D. Kiriya, D.-H. Lien, G. A. Brooks, R. W. Davis, A. Javey, Nature 2016, 529, 509 .

[27] L. Ortega, A. Llorella, J. P. Esquivel, N. Sabaté, Microsystems Nanoeng. 2019, 5, 3.

[28] D. H. Kim, J. Xiao, J. Song, Y. Huang, J. A. Rogers, Stretchable, curvilinear electronics based on inorganic materials. Adv. Mater. 2010, 22, 2108-2124.

[29] D. H. Kim, J. A. Rogers, Adv. Mater. 2008, 20, 4887.

[30] S. P. Lacour, J. Jones, Z. Suo, S. Wagner, Design and performance of thin metal film interconnects for skin-like electronic circuits. IEEE Electron Device Lett. 2004, 25, $179-181$.

[31] S.-I. Park, J.-H. Ahn, X. Feng, S. Wang, Y. Huang, J. A. Rogers, Adv. Funct. Mater. 2008, 18, 2673.

[32] A. C. Cavazos Sepulveda, M. S. Diaz Cordero, A. A. A. Carreño, J. M. Nassar, M. M. Hussain, Appl. Phys. Lett. 2017, 110, 134103.

[33] B. Huyghe, H. Rogier, J. Vanfleteren, F. Axisa, IEEE Trans. Adv. Packag. 2008, 31, 802.

[34] M. Gonzalez, F. Axisa, M. Vanden Bulcke, D. Brosteaux, B. Vandevelde, J. Vanfleteren, Microelectron. Reliab. 2008, 48, 825.

[35] J. A. Fan, W.-H. Yeo, Y. Su, Y. Hattori, W. Lee, S.-Y. Jung, Y. Zhang, Z. Liu, H. Cheng, L. Falgout, M. Bajema, T. Coleman, D. Gregoire, R. J. Larsen, Y. Huang, J. A. 


\section{WILEY-VCH}

Rogers, Nat. Commun. 2014, 5, 3266.

[36] K. L. Lin, K. Jain, IEEE Electron Device Lett. 2009, 30, 14.

[37] A. M. Hussain, E. B. Lizardo, G. A. Torres Sevilla, J. M. Nassar, M. M. Hussain, Adv. Healthc. Mater. 2015, 4, 665.

[38] N. Qaiser, S. M. Khan, M. M. Hussain, J. Appl. Phys. 2018, 124, 034905.

[39] N. Qaiser, S. M. Khan, M. Nour, M. U. Rehman, J. P. Rojas, M. M. Hussain, Appl. Phys. Lett. 2017, 111, 214102.

[40] A. M. Hussain, M. M. Hussain, In ASME International Mechanical Engineering Congress and Exposition, Proceedings (IMECE); American Society of Mechanical Engineers (ASME), 2015; Vol. 14-2015.

[41] A. N. Damdam, N. Qaisar, M. M. Hussain, Appl. Phys. Lett. 2019, 115, 112105.

[42] T. Widlund, S. Yang, Y.-Y. Hsu, N. Lu, Int. J. Solids Struct. 2014, 51, 4026.

[43] M. U. Rehman, J. P. Rojas, Extrem. Mech. Lett. 2017, 15, 44.

[44] K. J. Yu, Z. Yan, M. Han, J. A. Rogers, npj Flex. Electron. 2017, 1.

[45] J. P. Rojas, A. Arevalo, I. G. Foulds, M. M. Hussain, Appl. Phys. Lett. 2014, 105, 154101.

[46] N. Qaiser, A. N. Damdam, S. M. Khan, S. F. Shaikh, M. M. Hussain, Appl. Phys. Lett. 2019, 115 .

[47] Y. H. Jung, H. Zhang, S. J. Cho, Z. Ma, IEEE Trans. Electron Devices 2017, 64, 1881.

[48] R. H. Kim, M. H. Bae, D. G. Kim, H. Cheng, B. H. Kim, D. H. Kim, M. Li, J. Wu, F. Du, H. S. Kim, S. Kim, D. Estrada, S. W. Hong, Y. Huang, E. Pop, J. A. Rogers, Nano Lett. 2011, 11, 3881.

[49] J. Jones, S. P. Lacour, S. Wagner, Z. Suo, J. Vac. Sci. Technol. A Vacuum, Surfaces, Film. 2004, 22, 1723.

[50] N. Qaiser, Y. J. Kim, C. S. Hong, S. M. Han, J. Phys. Chem. C 2016, 120, 6953.

[51] S. Chugh, M. Man, Z. Chen, K. J. Webb, Appl. Phys. Lett. 2015, 106, 061102. 


\section{WILEY-VCH}

[52] X. L. Li, J. G. Guo, J. Nanomater. 2019, 9734807.

[53] H. Ogawa, K. Suzuki, S. Kaneko, Y. Nakano, Y. Ishikawa, T. Kitahara, Microsyst. Technol. 1997, 3, 117.

[54] D. Kim, S. H. Kim, J. H. Kim, J. C. Lee, J. P. Ahn, S. W. Kim, Sci. Rep. 2017, 7.

[55] N. André, M. Coulombier, V. De Longueville, D. Fabrègue, T. Gets, S. Gravier, T. Pardoen, J. P. Raskin, Microelectron. Eng. 2007, 84, 2714.

[56] S. Olliges, P. A. Gruber, V. Auzelyte, Y. Ekinci, H. H. Solak, R. Spolenak, Acta Mater. 2007, 55, 5201.

[57] C. S. Hong, N. Qaiser, H. G. Nam, S. M. Han, Phys. Chem. Chem. Phys. 2019, 21, 9581. 


\section{WILEY-VCH}

(a) Spiral

$L_{\mathrm{a}}$ of the spiral $=2.683 \mathrm{~mm}$

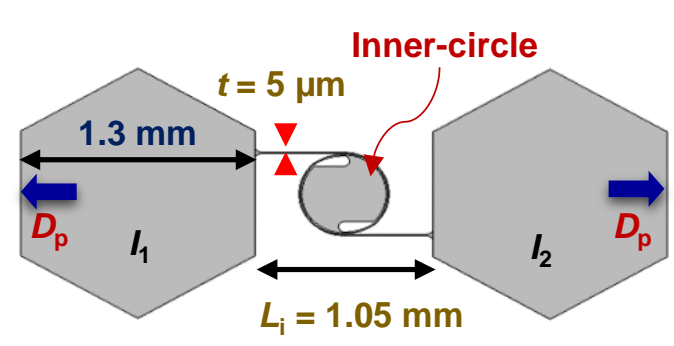

(b) Horseshoe

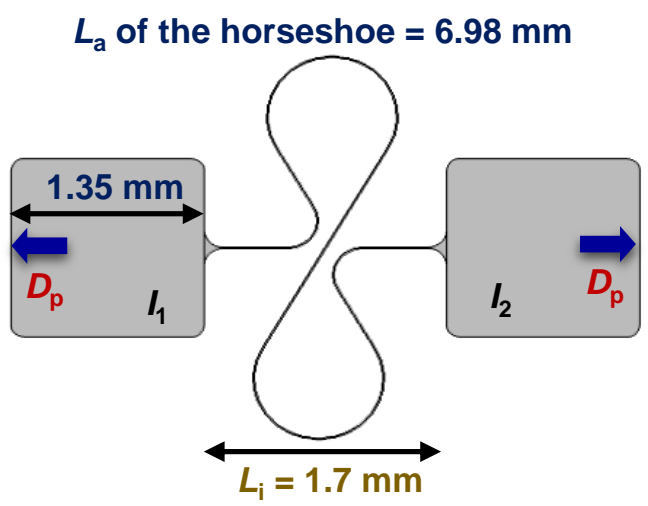

Fig. 1: Schematics of the spiral and horseshoe and associated parameters for stretchable island-interconnection configurations. (a) Spiral and (b) horseshoe interconnections connected to the islands. The initial length $\left(L_{\mathrm{i}}\right)$ of interconnections corresponds to the gap between two islands $\left(I_{1}\right.$ and $\left.I_{2}\right)$ at undeformed states, whereas the arc length $\left(L_{\mathrm{a}}\right)$ can be regarded as the actual length of interconnection or gap between two islands when a given interconnection is at a fully stretched state, and it becomes rectilinear. Arrows show the direction of in-plane prescribed displacement, i.e., $D_{\mathrm{p}}$. 


\section{WILEY-VCH}

(a)

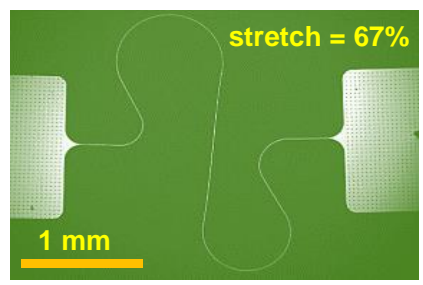

(d)

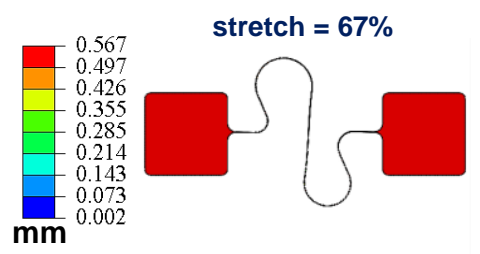

(b)

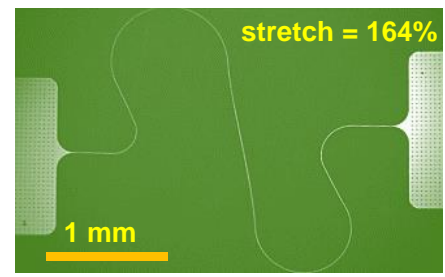

(e)

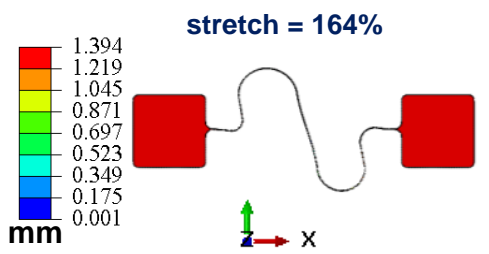

(c)

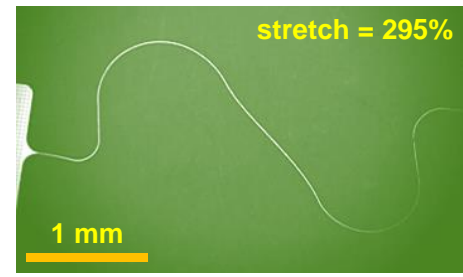

(f) (g) Stretch $=408 \%$

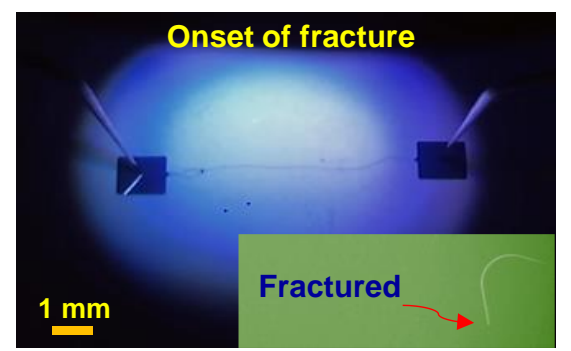

(i) von Mises stress ( $\sigma$ )

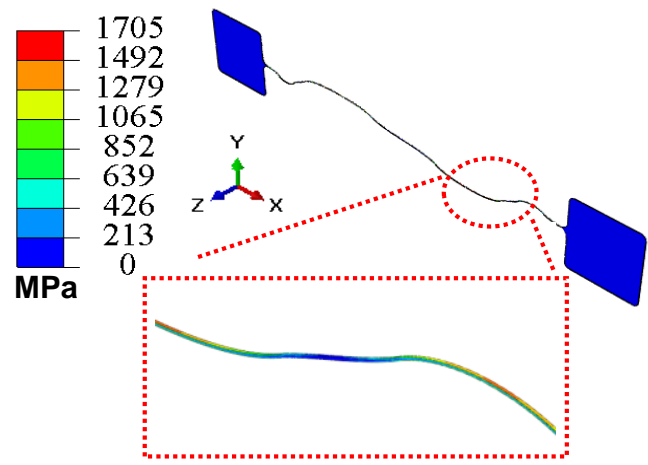

(h) FEM stretch $=408 \%$

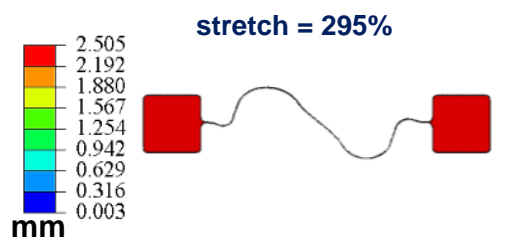

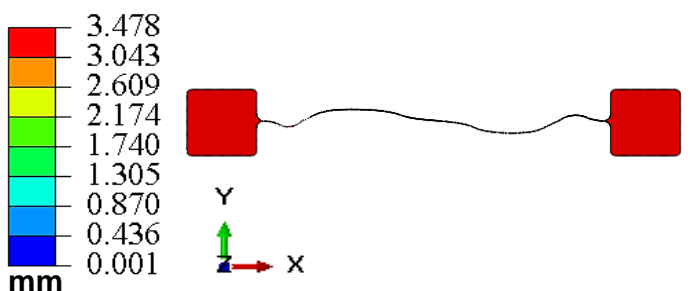

(j) Principal strain

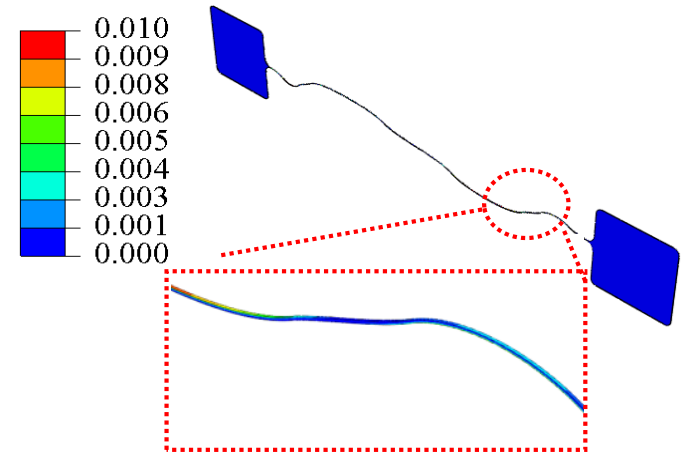

Figure 2. Mechanical response and failure criteria of the horseshoe by experimental and FEM analysis. (a-c) Stretching profiles of the fabricated interconnection with a $t$ of $5 \mu \mathrm{m}$ from the undeformed state to applied stretch of $67 \%, 164 \%$, and $295 \%$, respectively. (d-f) FEM shows the deformations for the same amount of stretching to corroborate with the experimental findings. (g) The optical image at the onset of the fracture, which reveals a maximum possible stretch of $408 \%$. Meanwhile, the inset shows the states of the broken horseshoe after the stretch exceeds $408 \%$. The FEM for the same amount of stretching shows the (h) deformation contours, and the $(i, j)$ von Mises stress and principal strain contours, respectively. The stress and strain states are essential to reveal the region that is prone to failure. It is clear from the results that the principal strain reaches $1 \%$ for $\mathrm{Si}$ with a corresponding stretch of $408 \%$, i.e., the maximum stretchability that is attained by a horseshoe with a $t$ of $5 \mu \mathrm{m}$. The insets of figure $2 \mathrm{i}$ and $2 \mathrm{j}$ show the zoomed images of the stress and strain contours. 


\section{WILEY-VCH}

(a)

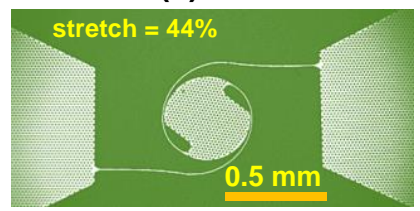

(d)

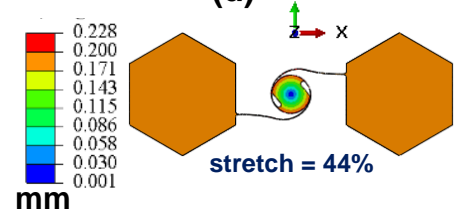

(g) Stretch $=154 \%$

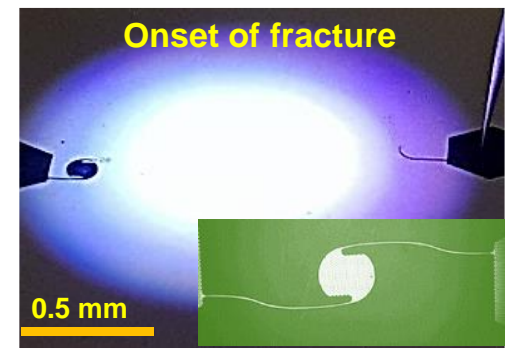

(i) von Mises stress $(\sigma)$

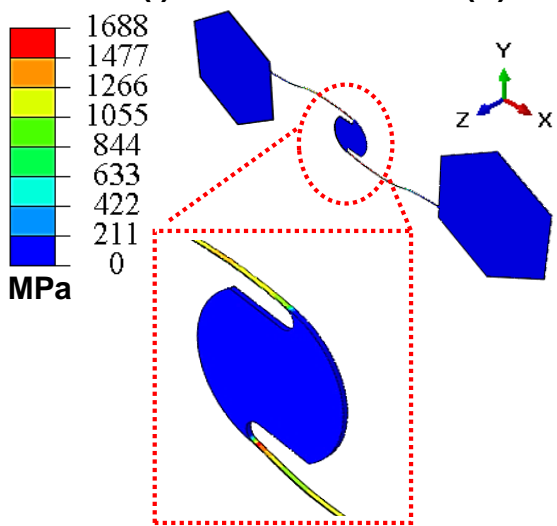

(b)

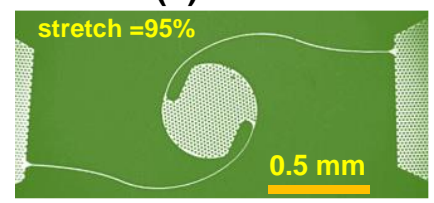

(e)
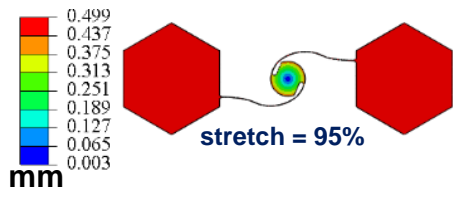

(c)

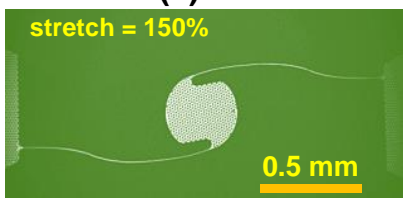

(f)

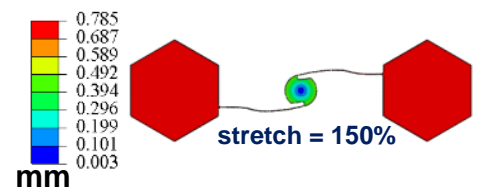

(h) FEM stretch $=154 \%$

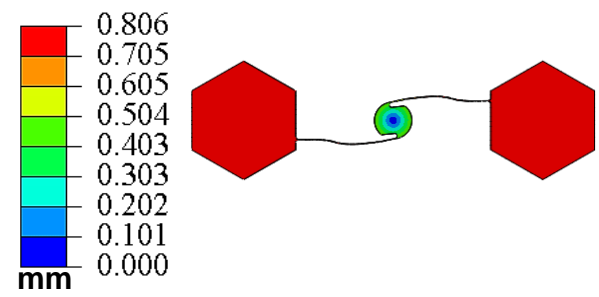

\section{(j) Principal strain}

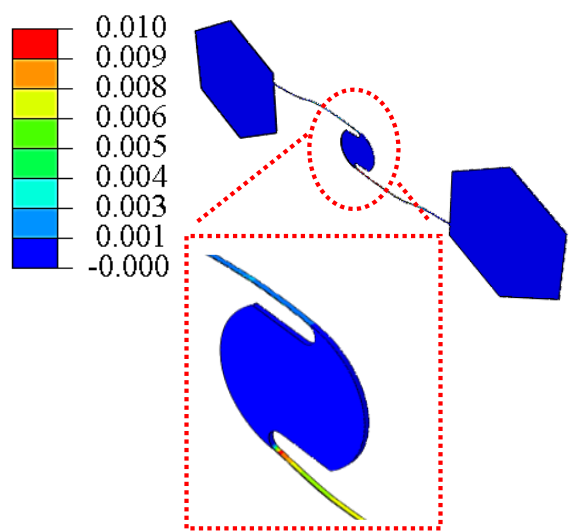

Figure 3. Mechanical response and failure criteria of the spiral by experimental and FEM analysis. (a-c) Stretching profiles of the fabricated interconnection with a $t$ of $5 \mu \mathrm{m}$, from the initial state to $44 \%, 95 \%$, and $150 \%$ of the stretch, respectively. (d-f) Numerical calculations show the deformation contours to corroborate with the same experimental stretch. (g) Optical image shows the maximum stretch of $154 \%$ for the fabricated interconnection when it exceeds the fracture conditions. The inset shows the state of the spiral before it breaks. For the same amount of stretching, the numerical calculation shows the (h) deformation contours, (i), von Mises stress, and (j) principal strain distributions. Results clarify that the principal strain exceeds the strain of $1 \%$ for the corresponding von Mises stress value of $1.69 \mathrm{GPa}$ when the structure experiences a fracture. The calculated stress of $1.69 \mathrm{GPa}$ is almost equal to the predicted value of the fracture stress from Equation 2. The insets of Figures $3 i$ and $3 j$ show the zoomed images of the stress and strain contours. 
(a) $\downarrow<\mathrm{t}$
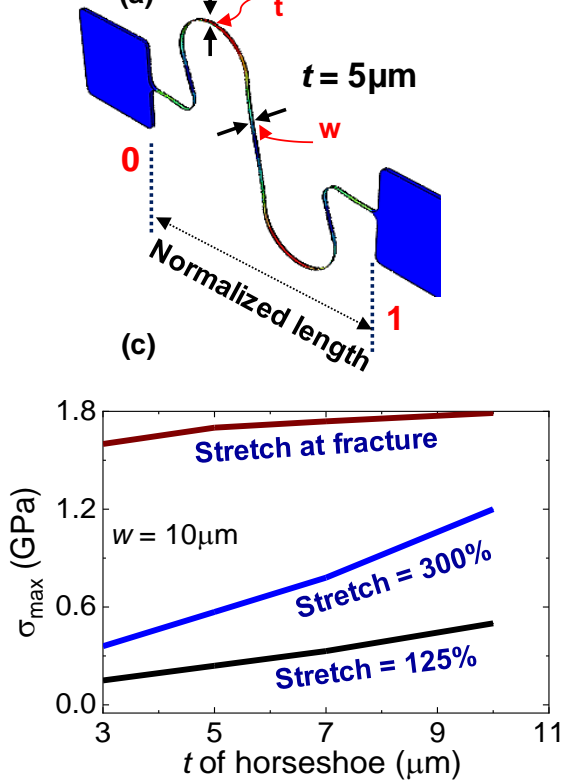

(e)

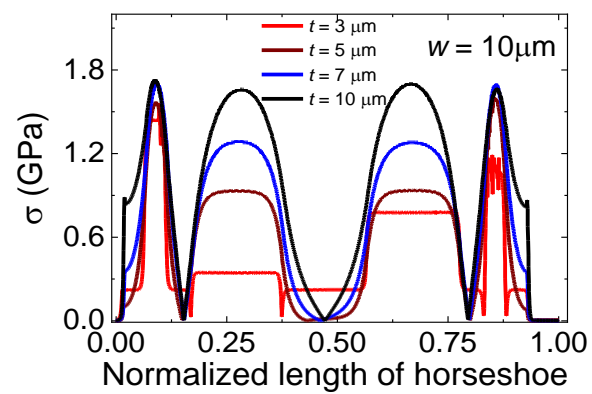

(g)

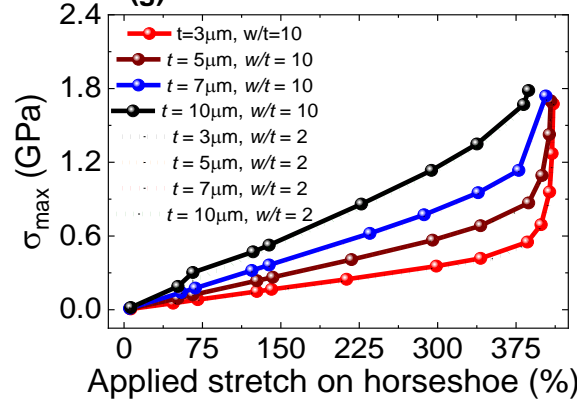

(b)
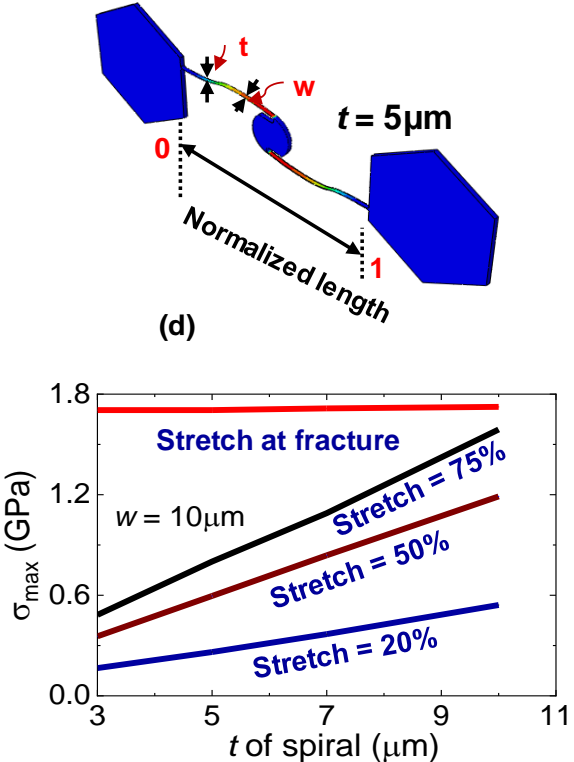

(f)

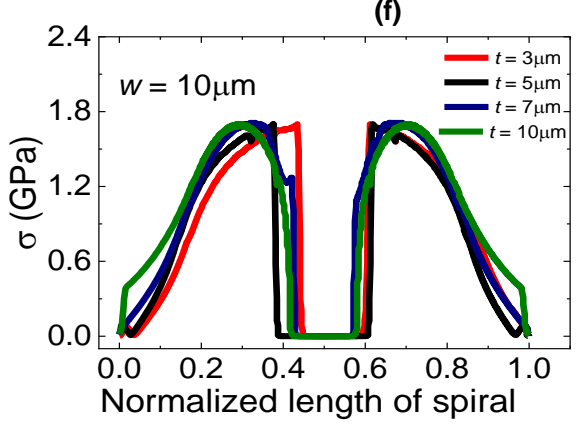

(h)

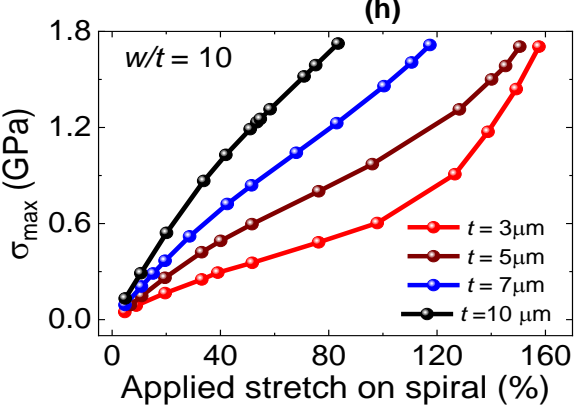

Figure 4. Design criteria for the horseshoe and spiral structures. (a, b) Representative designs of the horseshoe and spiral in stretched form along with the definition of associated parameters. For the horseshoe and spiral, (c, d) the maximum von Mises stress as a function of $t$ (i.e., $3 \mu \mathrm{m}$ to $10 \mu \mathrm{m}$ ) during the various stages of stretching until it experiences a fracture. During earlier stages of stretching, the stress linearly changes with the $t$ of the arm. However, regardless of the $t$ of the arm, the stress is saturated once the spiral arm attains the fracture conditions. (e, f) For the various sizes of $t$ for the horseshoe and spiral arm, the evolution of stress along the normalized length, this shows the region of the stress concentration. $(\mathrm{g})$ Maximum stress distribution for the different parameters when the horseshoe arm is stretched from the initial state to the failure state, i.e., the range of the applied stretch. The results show that the thin interconnection has the lowest stress evolution, which is up to $90 \%$ of its maximum stretchability; however, the stress is almost linear for a thicker counterpart. In addition, the (h) maximum stress distribution of a spiral arm is a function of the applied stretch, i.e., from $0 \%$ to $154 \%$. By contrast, the spiral starts experiencing stress during the earlier stages of stretching. 
(a)

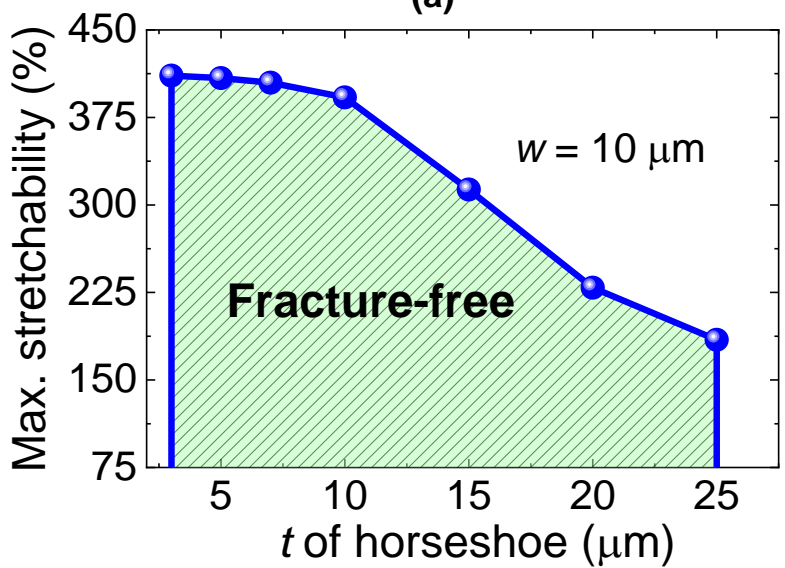

(c)

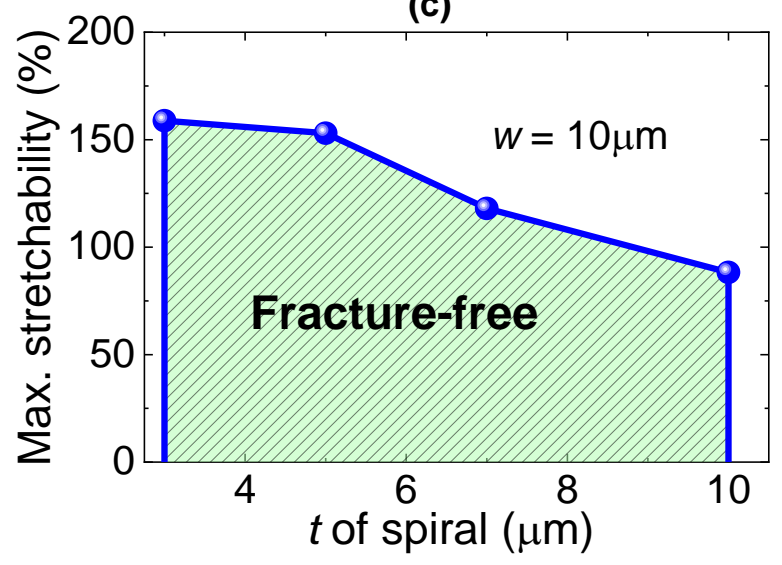

(e)

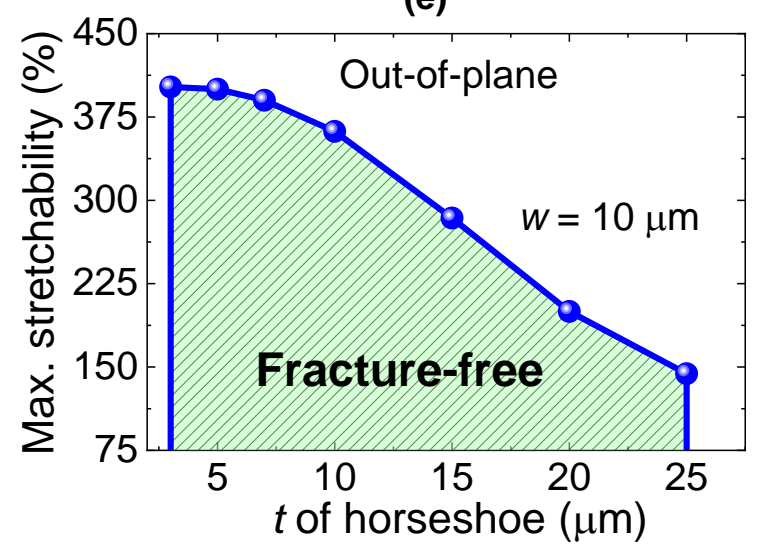

(b)

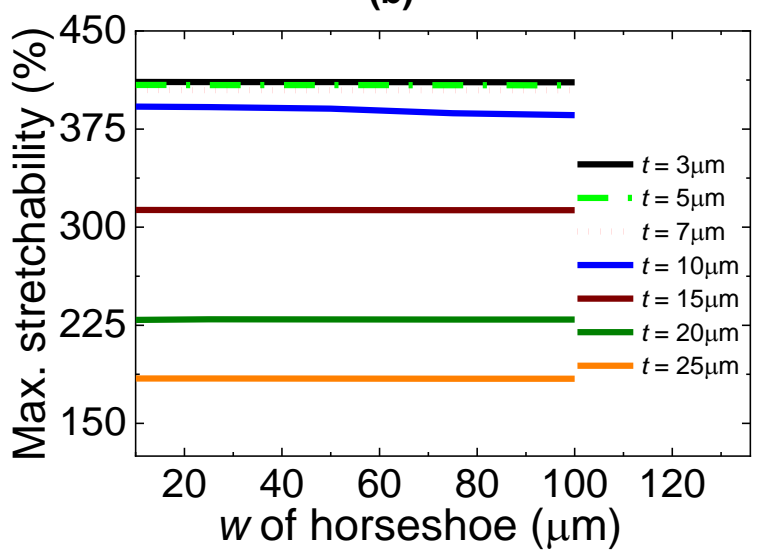

(d)

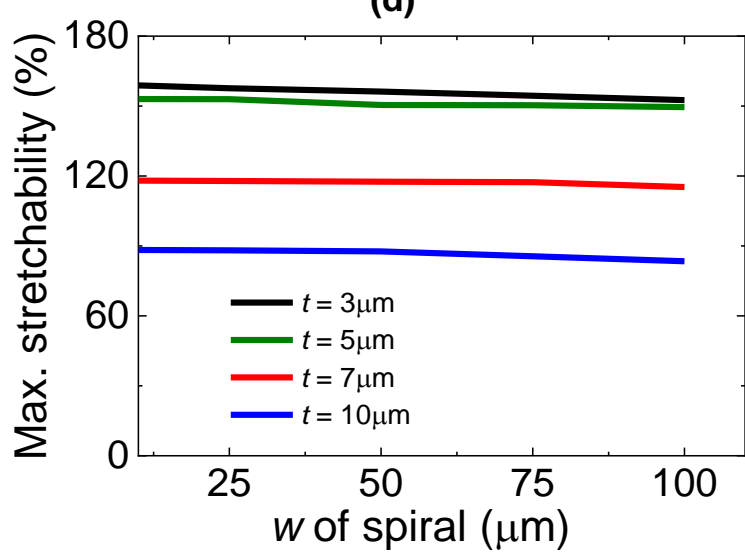

(f)

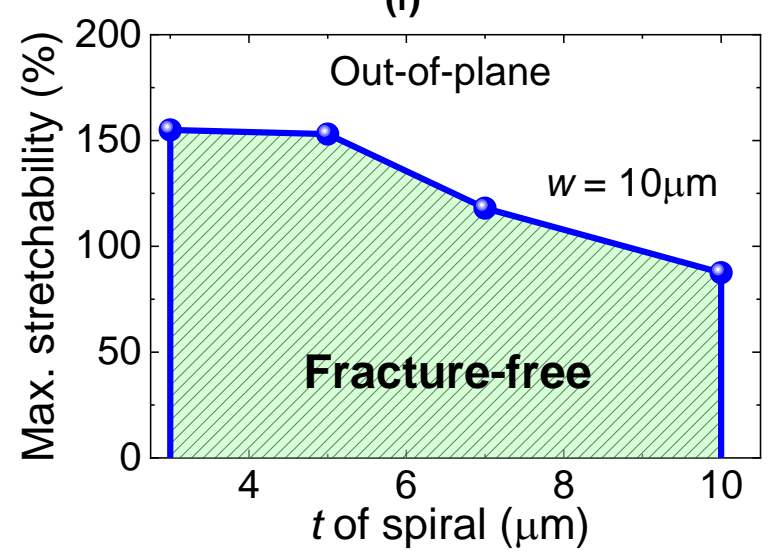

Figure 5. Figure-of-merit in terms of stretchability for the horseshoe and spiral. (a, c) Maximum stretchability for the horseshoe and spiral for a wide range of $t$ and constant values of $w$. We take the cutoff value of $t$ once the stretchability of the interconnection is reduced to $50 \%$. The area under the curve provides the fracture-free region, that is, the interconnection will not fail and will be reversible. (b, d) Stretchability of the horseshoe and spiral as a function of $w$ shows the insignificant effect of $w$. The results show that the width has negligible effects, whereas $t$ has a pronounced impact on the mechanical integrity of the interconnections. The out-of-plane response for $(\mathrm{e}, \mathrm{f})$ the horseshoe and spiral has stretchability that is comparable to in-plane stretching. For the horseshoe, due to out-of-plane twisting, the stretchability was reduced to $144 \%$ for a thicker interconnection (i.e., $t=25 \mu \mathrm{m}$ ). 


\section{WILEY-VCH}

(a) Stretch $=183 \%$

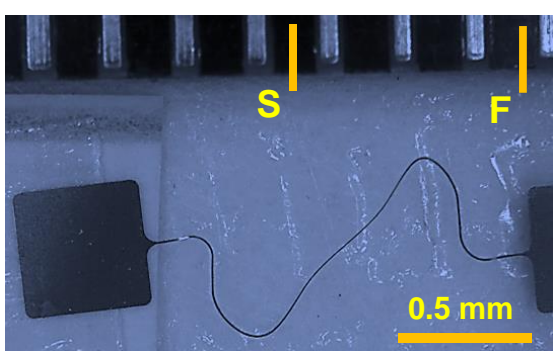

$t=25 \mu \mathrm{m}, w=10 \mu \mathrm{m}$

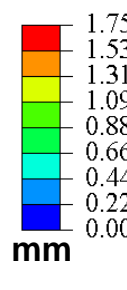

(d) Stretch $=90 \%$
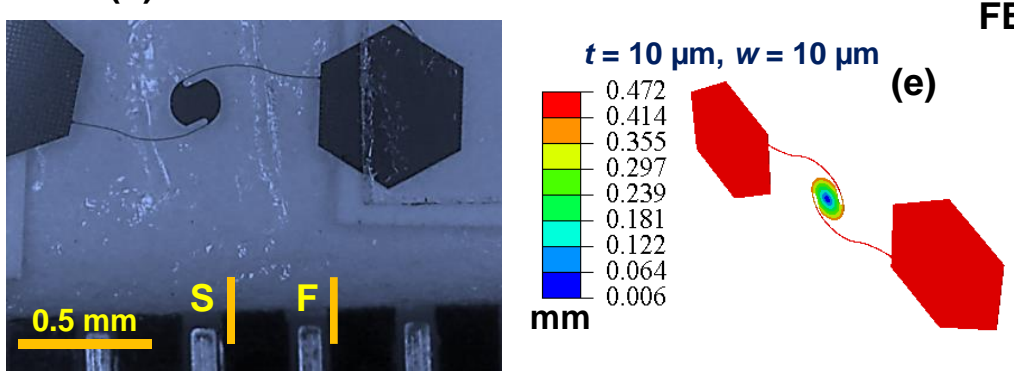

FEM stretch $=90 \%$ (b)

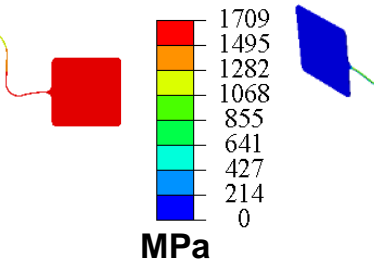

(c)

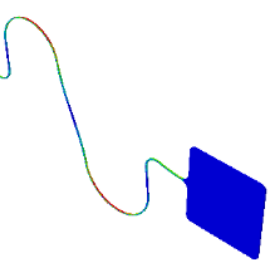

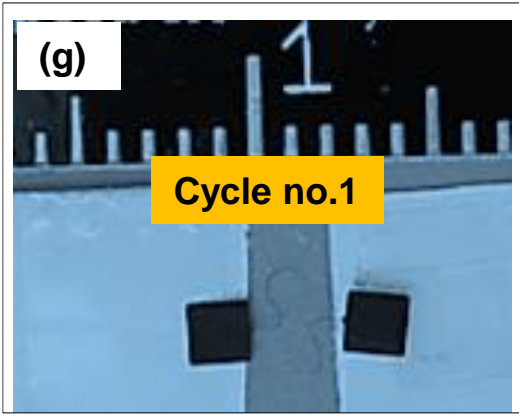
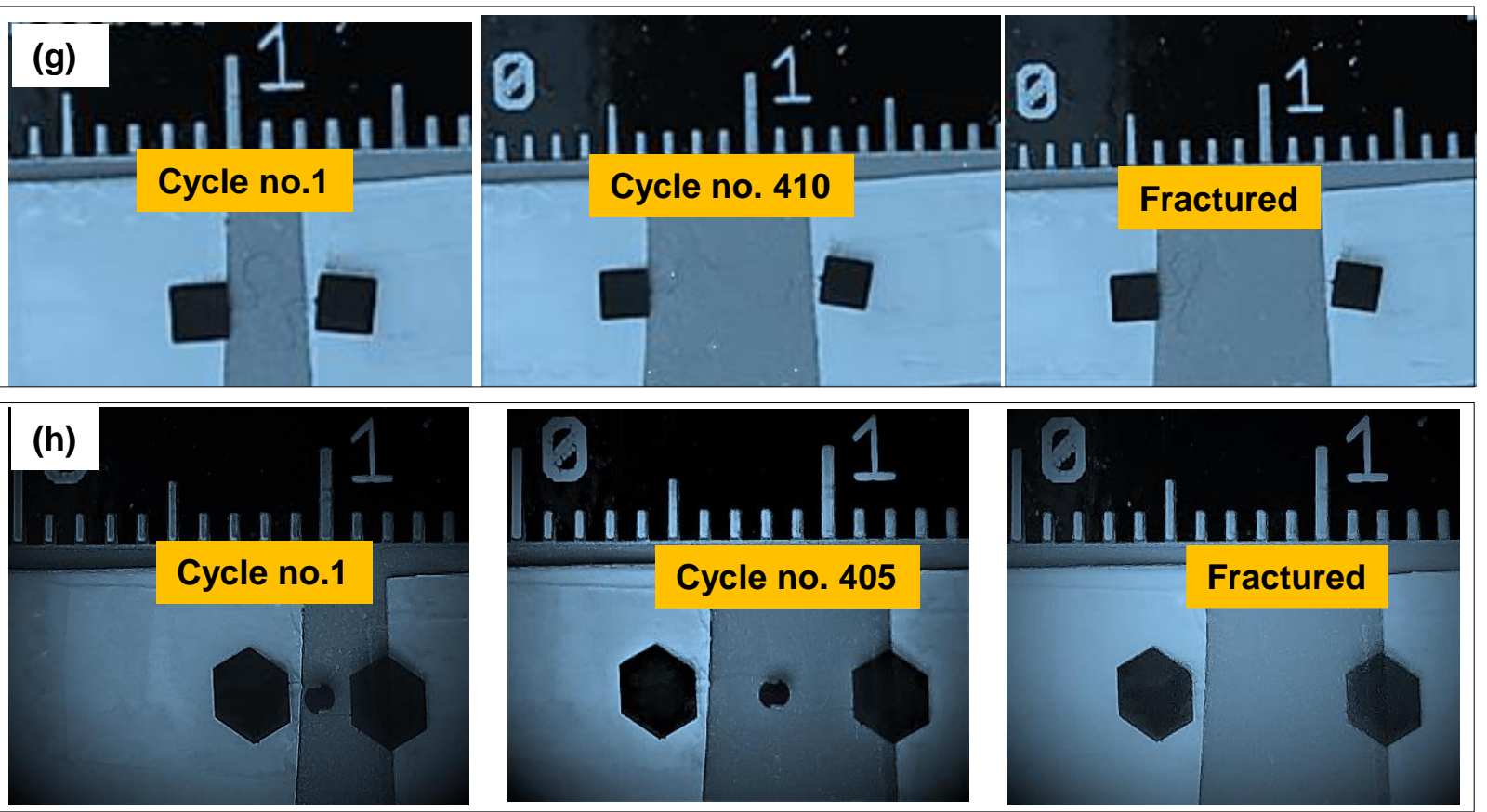

Figure 6. Cyclic life and failure criteria of the horseshoe and spiral with $\boldsymbol{t}$ values of $\mathbf{2 5} \boldsymbol{\mu m}$ and $10 \mu \mathrm{m}$. For the horseshoe, (a-c) the experimental stretching profiles, FEM-based deformation, and von Mises stress contours for the applied stretch of $183 \%$, i.e., on the verge of the fracture. For the spiral, (d-f) the experimental stretching profile, FEM-based deformation contour, and von Mises stress contours with an applied stretch of $90 \%$, i.e., on the verge of the fracture. The letters $S$ and $F$ represent the start and finish positions of the stretch. Cyclic life of (g) horseshoe and (h) spiral shows that both structures have a higher cyclic life, i.e., greater than 400 cycles. 
(a)

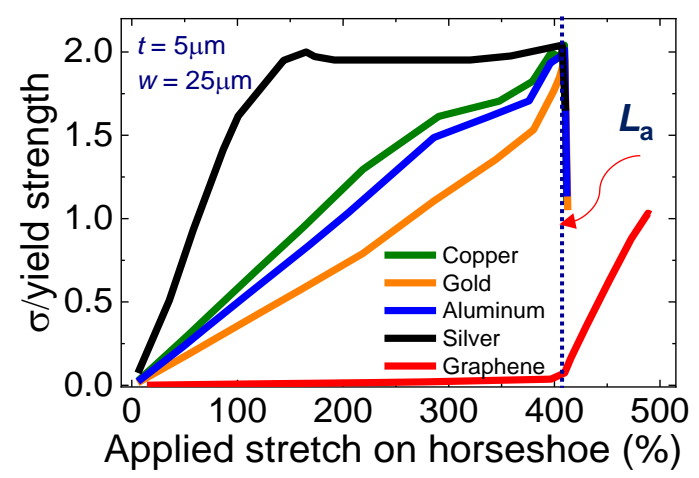

(c)

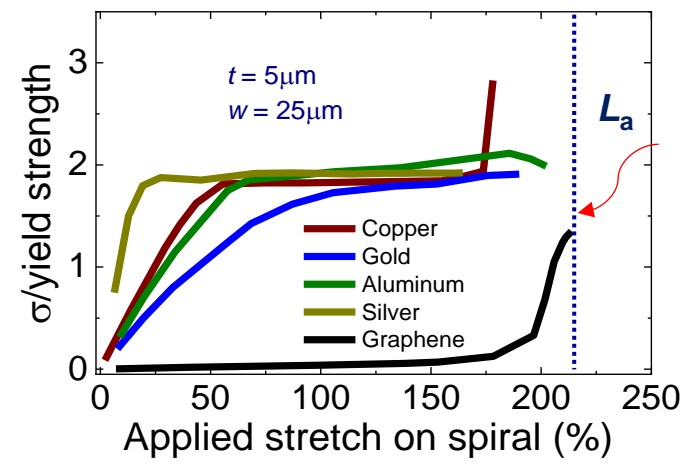

(e)

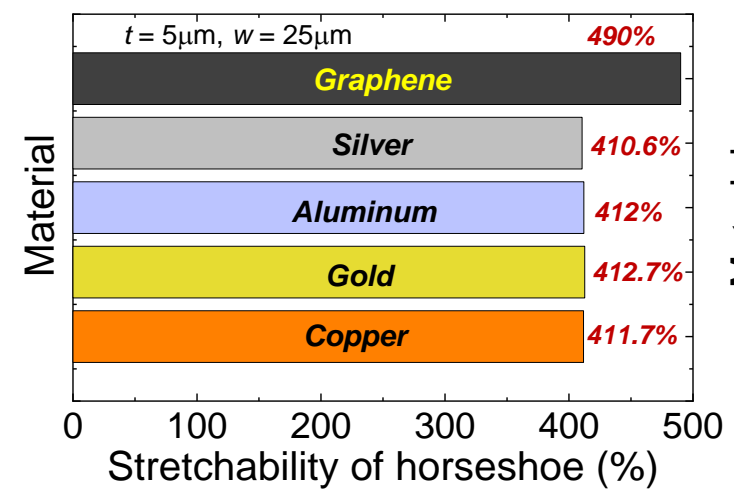

(b)

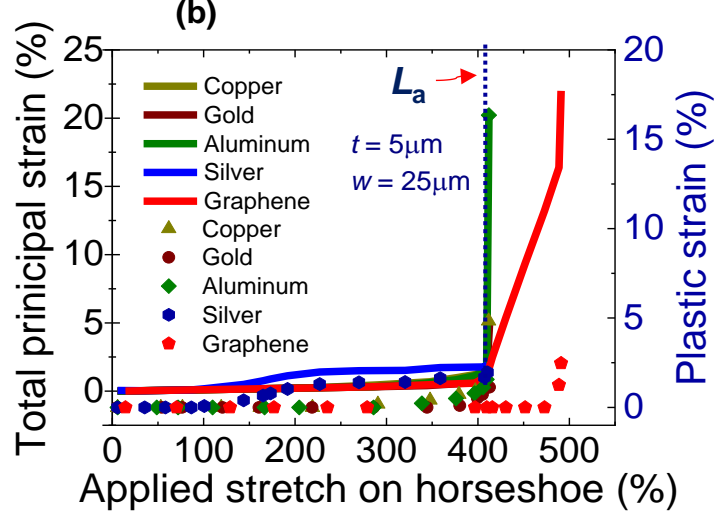

(d)

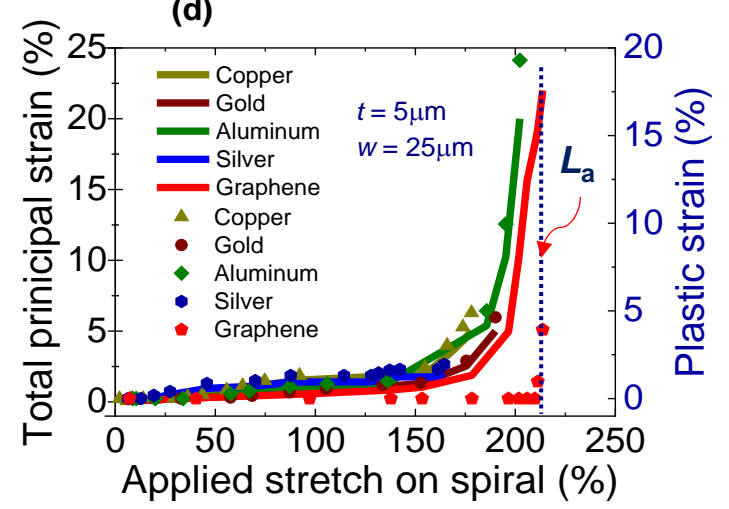

(f)

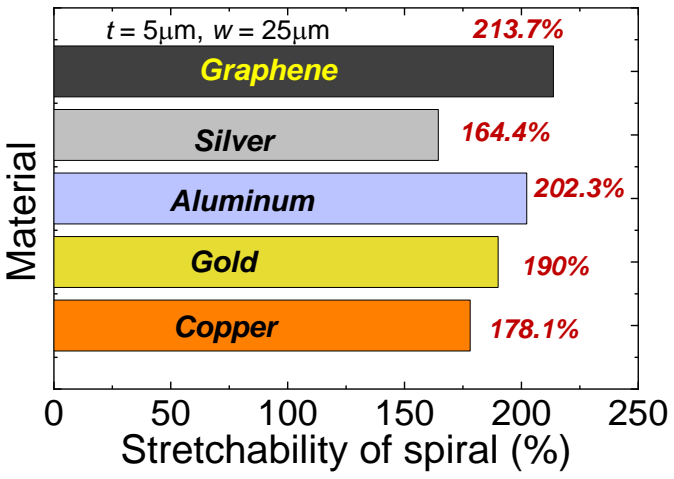

Figure 7. Design perspective and stretchability for the horseshoe and spiral $(t=5 \boldsymbol{m}$ and $\boldsymbol{w}=\mathbf{2 5} \boldsymbol{\mu m}$ ) for a variety of materials. (a, c) Normalized stress, i.e., von Mises stress/yield strength as a function of applied stretch on the horseshoe and spiral arm. Results show that silver undergoes a higher stress even during an earlier stage of stretching. By contrast, graphene has a low stress until it reaches $90 \%$ of its stretch. $L_{\mathrm{a}}$ shows the point where the interconnection opens up to its arc length. i.e., a further stretch will thin down the interconnection itself. (b, d) On the same magnitude of the applied stretch, the evolution of the total principal and plastic strain for all of the materials of the horseshoe and spiral arm. Stretchability of interconnections for (e) horseshoe and (f) spiral. Results reveal that graphene has a higher stretchability in comparison with other materials. 


\section{WILEY-VCH}

Table 1. Design guidelines for the horseshoe and spiral that exhibit the maximum allowable stretchability and fracture stress. For a constant $t$ of $5 \mu \mathrm{m}$, the intrinsic fracture stain and prescribed displacements cause the failure of the respective design and materials.

\begin{tabular}{|c|c|c|c|c|c|c|}
\hline $\begin{array}{c}\text { Interconnection } \\
\text { Type }\end{array}$ & Material & $\begin{array}{l}\text { Thickness } \\
\qquad(\mu \mathrm{m})\end{array}$ & $\begin{array}{c}\text { Prescribed } \\
\text { Displacement } \\
(\mathrm{mm})\end{array}$ & $\begin{array}{c}\text { Maximum } \\
\text { Stretchability } \\
(\%)\end{array}$ & $\begin{array}{c}\text { Intrinsic } \\
\text { Fracture } \\
\text { Strain } \\
(\%)\end{array}$ & $\begin{array}{l}\text { Stress } \\
\text { at } \\
\text { Fracture } \\
\text { (GPa) }\end{array}$ \\
\hline \multirow[t]{6}{*}{ Horseshoe } & Silicon & 5 & 6.98 & 410.59 & 1 & 1.70 \\
\hline & Silver & 5 & 6.98 & 410.59 & 2 & 0.089 \\
\hline & Gold & 5 & 6.99 & 411.17 & 5 & 0.22 \\
\hline & Copper & 5 & 7.0 & 411.76 & 5 & 0.24 \\
\hline & Aluminum & 5 & 7.0 & 411.76 & 20 & 0.17 \\
\hline & Graphene & 5 & 8.346 & 490.94 & 22 & 153 \\
\hline \multirow[t]{6}{*}{ Spiral } & Silicon & 5 & 1.612 & 154 & 1 & 1.69 \\
\hline & Silver & 5 & 1.726 & 164.44 & 2 & 0.104 \\
\hline & Gold & 5 & 2.0 & 190 & 5 & 0.401 \\
\hline & Copper & 5 & 1.8702 & 178.11 & 5 & 0.622 \\
\hline & Aluminum & 5 & 2.124 & 202.28 & 20 & 0.298 \\
\hline & Graphene & 5 & 2.244 & 213.71 & 22 & 202 \\
\hline
\end{tabular}




\section{WILEY-VCH}

\section{Table of Contents (TOC)}

The physical significance of the horseshoe and spiral topology-based stretchable devices reveals how their parameters control the mechanical integrity without changing the areal efficiency. The results indicate why a particular material with a given interconnection size would fail and will not avert the fracture. The results prove that the thickness of the interconnections plays a crucial role, and the width has an insignificant effect. The figure-ofmerit, in terms of the stretchability, shows optimized topologies for high-performance stretchable electronics.
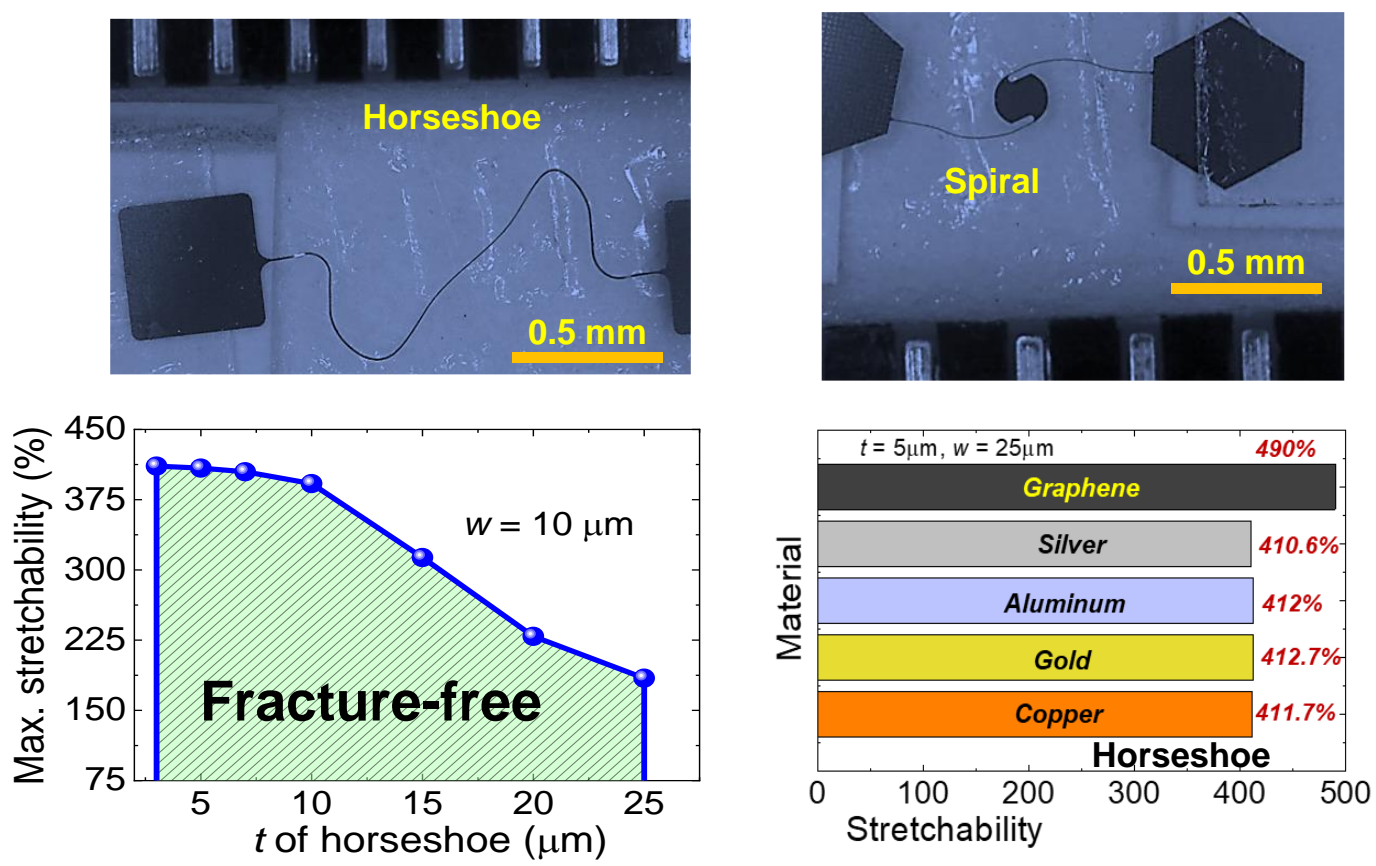

Figure 1 Figure-of-merit for Si horseshoe and spiral, and stretchability for commonly used materials in stretchable electronics. 


\section{Supporting Information}

\section{Design Criteria for Horseshoe and Spiral-based Interconnects for Highly Stretchable Electronic Devices}

Dr. Nadeem Qaiser ${ }^{l}$, Asrar Nabil Damdam ${ }^{l}$, Dr. Sherjeel Munsif Khan ${ }^{l}$, Saleh Bunaiyan ${ }^{1}$, Prof. Muhammad Mustafa Hussain ${ }^{1},{ }^{2 *}$

${ }^{1}$ mmh Labs, Electrical Engineering, Computer Electrical and Mathematical Science and Engineering Division, King Abdullah University of Science and Technology (KAUST),

Thuwal 23955, Saudi Arabia, Tel.: +966-544-700-072

${ }^{2}$ EECS, University of California, Berkeley, CA 94720, USA.

*Corresponding author; E-mail: mmhussain@berkeley.edu I

muhammadmustafa.hussain@kaust.edu.sa 


\section{WILEY-VCH}

1- Fabrication of interconnect-island configurations

(a) Si wafer

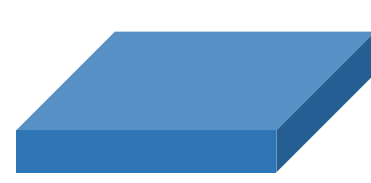

(f) Etching of Al

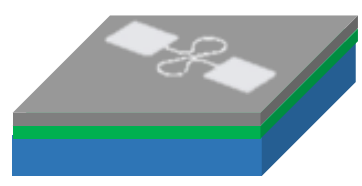

I (b) PECVD of $\mathrm{SiO}_{2}$

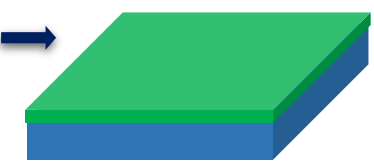

(e) Photolithography

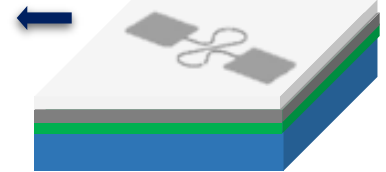

(h) Al Wet etching

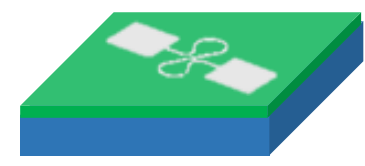

(c) PECVD of a-Si

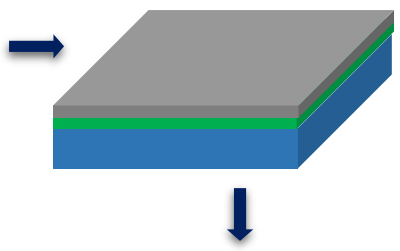

(d) Al sputtering

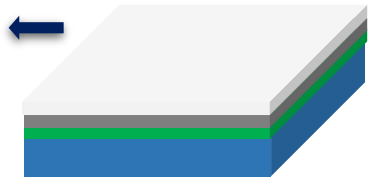

(i) HF etching
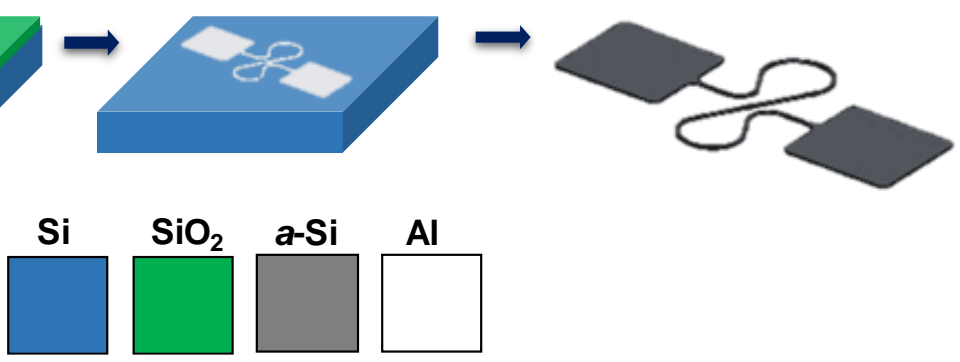

Figure S1. The fabrication process of the horseshoe and spiral interconnects-island configurations. (a) P-type bare Silicon (Si) wafer. (b, c) Oxide and amorphous $\mathrm{Si}(a-\mathrm{Si})$ of 10 $\mu \mathrm{m}$ each is deposited by using the PECVD process. The need for the hard mask is fulfilled by the (d) deposition of aluminum (Al) by using the sputtering. (e) Stretchable designs are patterned on the stack of $\mathrm{Al} / \mathrm{a}-\mathrm{Si} / \mathrm{SiO} 2 / \mathrm{Si}$ by using the photolithography. (f) $\mathrm{Al}$ is etched by the Reactive Ion Etching process (RIE). (g) The width of horseshoe and spiral structures is achieved by the deep RIE, i.e., down to the size of about $10 \mu \mathrm{m}$. (h) The remaining $\mathrm{Al}$ on top of the interconnect is etched by using the wet $\mathrm{Al}$ etchant. Finally, (i) release of interconnects is conducted by using the $48 \%$ HF (Hydrofluoric acid), i.e., wet isotropic etching step for 1 hour at room temperature. 


\section{WILEY-VCH}

2- Experimental setup and initial undeformed states of the fabricated samples

(a)

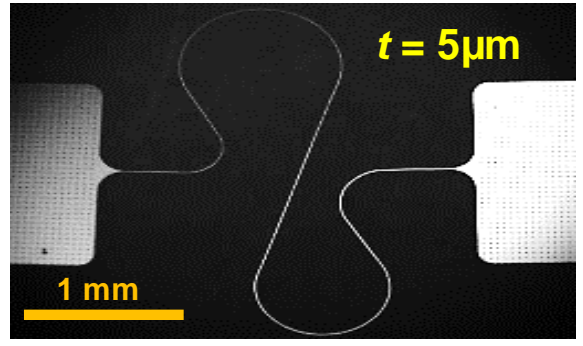

(c)

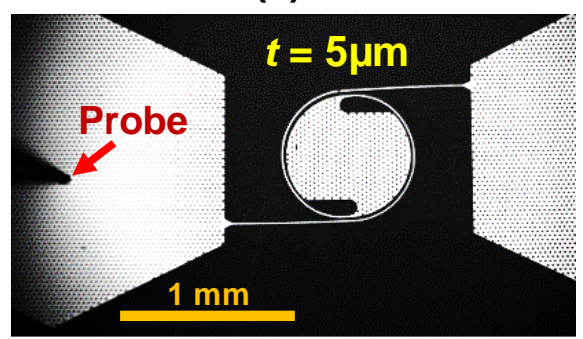

(b)

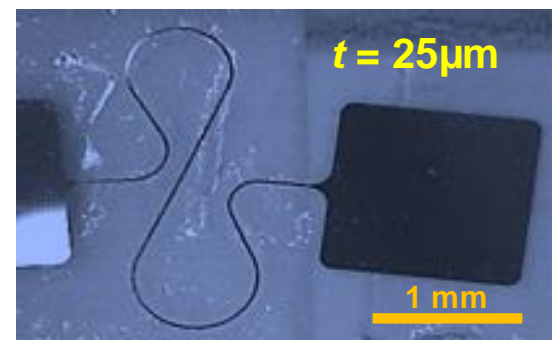

(d)

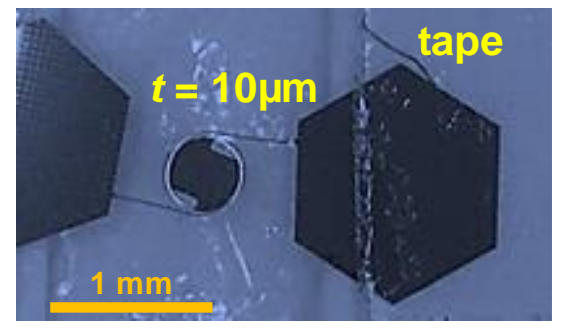

Figure S2. Optical images of initially undeformed shapes of fabricated structures. Horseshoe with a $t$ of (a) $5 \mu \mathrm{m}$ and (b) $25 \mu \mathrm{m}$. Spiral with $t$ of (c) $5 \mu \mathrm{m}$ and (d) $10 \mu \mathrm{m}$, respectively. The scotch tape was used to firmly hold the islands to eliminate the out-of-plane or rotational motions. The scale bar is shown in each figure. 


\section{WILEY-VCH}

3- Numerical calculations for 3D stress contours of horseshoe
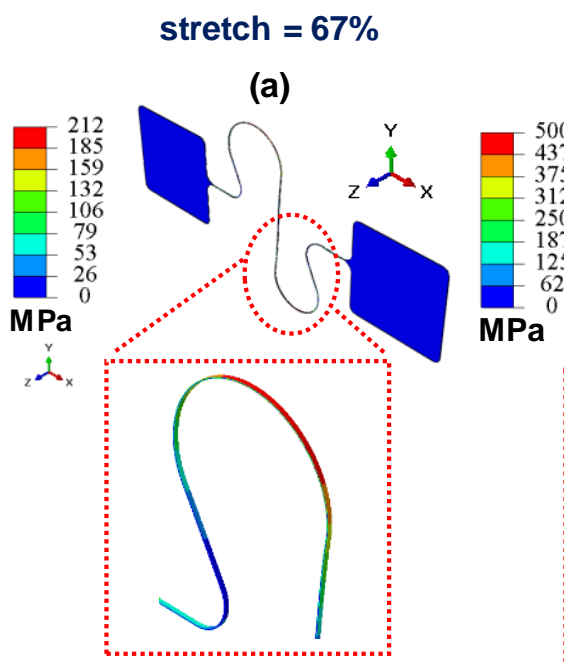

$$
\text { stretch }=164 \%
$$

(b)

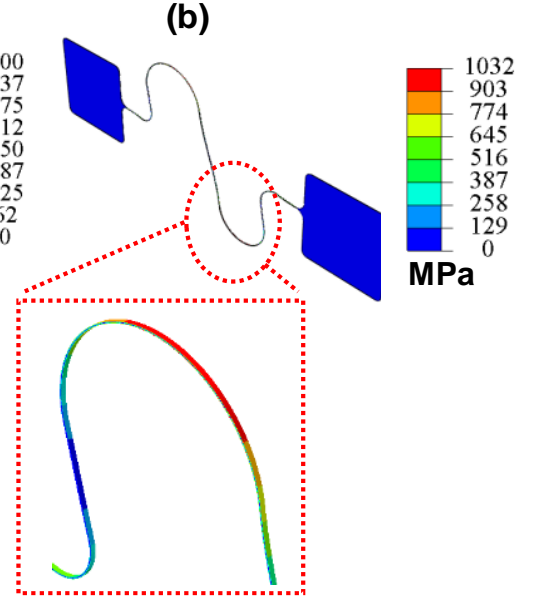

stretch $=295 \%$

(c)

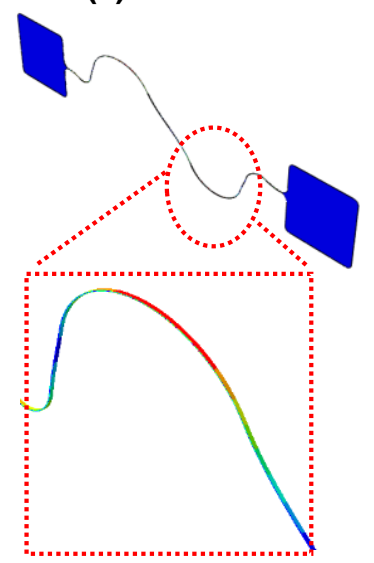

Figure S3. Distribution of von Mises stress in horseshoe by numerical calculations. (a-c) $3 \mathrm{D}$ contours at the applied stretch of $67 \%, 164 \%$, and $295 \%$, respectively. Magnified images are shown as the inset to reveal the stress contours along the arm of the horseshoe. Results show the region with stress-concentration, and upon the further stretch, it is expected to initiate the fracture in these regions. 


\section{WILEY-VCH}

4- Numerical calculations for 3D stress contours of spiral

(a) stretch $=44 \%$
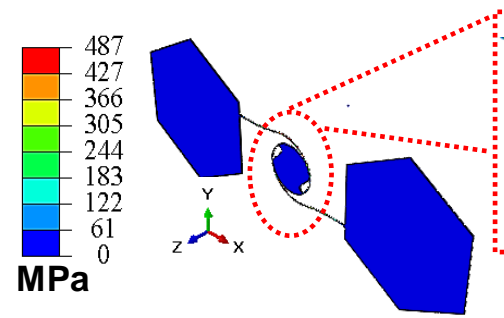

(c) stretch $=132 \%$

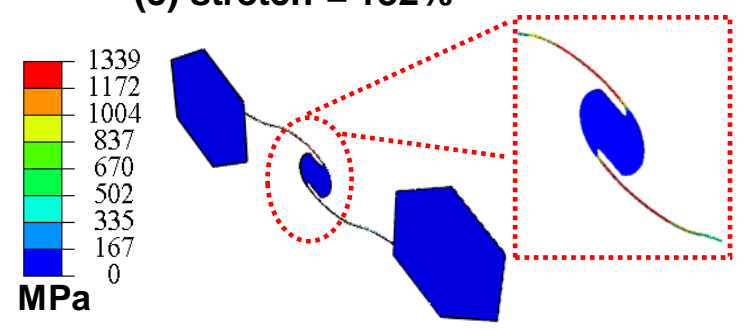

(b) stretch $=95 \%$

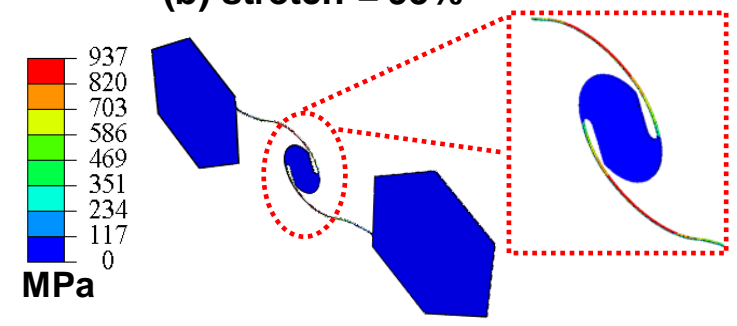

(d) stretch $=150 \%$

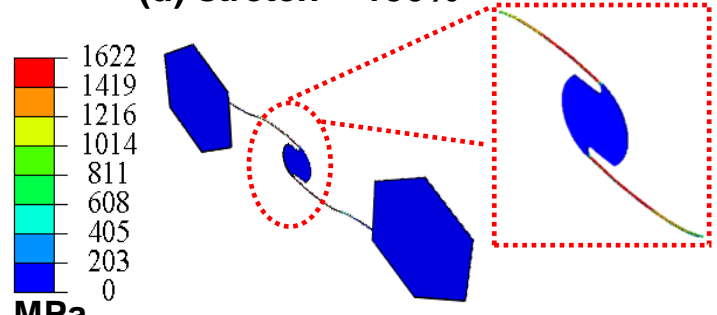

Figure S4. Distribution of von Mises stress in the spiral by numerical calculations. (a-d) $3 \mathrm{D}$ contours at the applied stretch of $44 \%, 95 \%, 132 \%$, and $150 \%$, respectively. Zoomed out images reveal the area with high stress or stress concentration during the spiral functioning. 
5- At failure, the evolution of deformations and strain for horseshoe
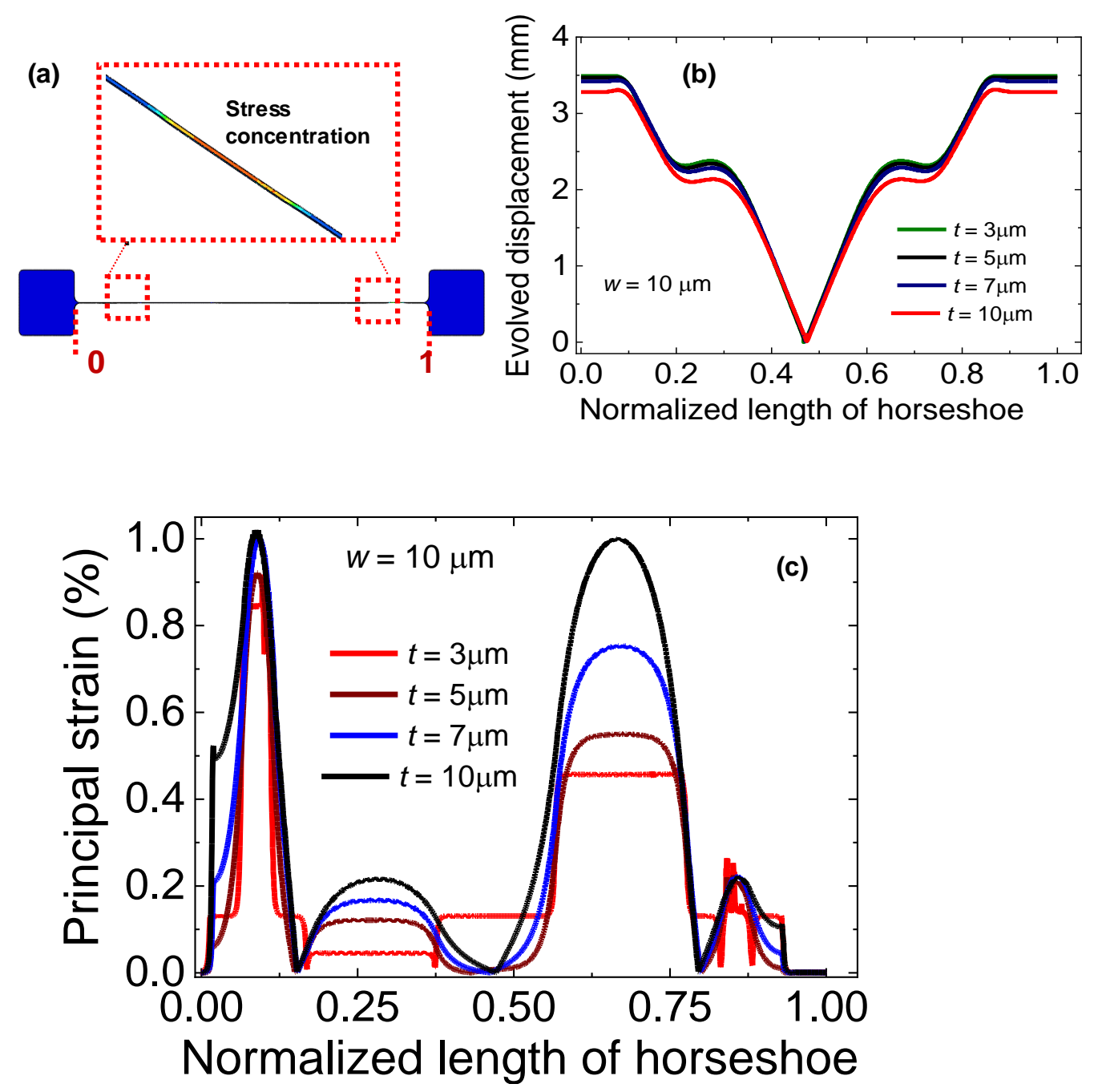

Figure S5. At fracture conditions, mechanical response along the arm of the horseshoe. (a) Fully stretched horseshoe of $t$ of $5 \mu \mathrm{m}$ showing the location of the stress concentration. The horseshoe's normalized length, along with positions of 0 and 1, are also shown in the figure. Evolution of (b) displacement and (c) principal strain along the normalized length of horseshoe arm, for $t$ of $3 \mu \mathrm{m}, 5 \mu \mathrm{m}, 7 \mu \mathrm{m}$, and $10 \mu \mathrm{m}$, respectively. It is interesting to observe that the experimental findings corroborate with the stress concentration region, i.e., interconnects experiences mechanical failure in these areas. 


\section{WILEY-VCH}

6- At failure, the evolution of deformations and stress/strain for spiral

(a)

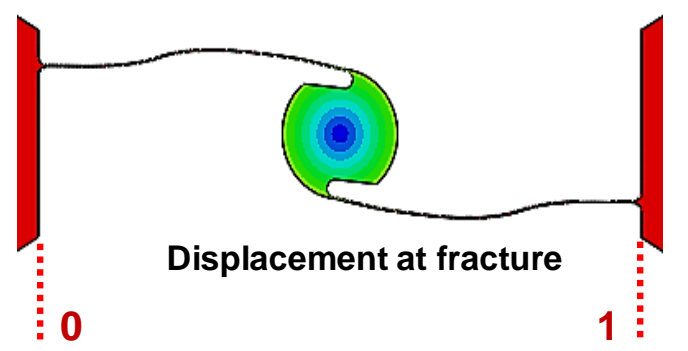

(b)

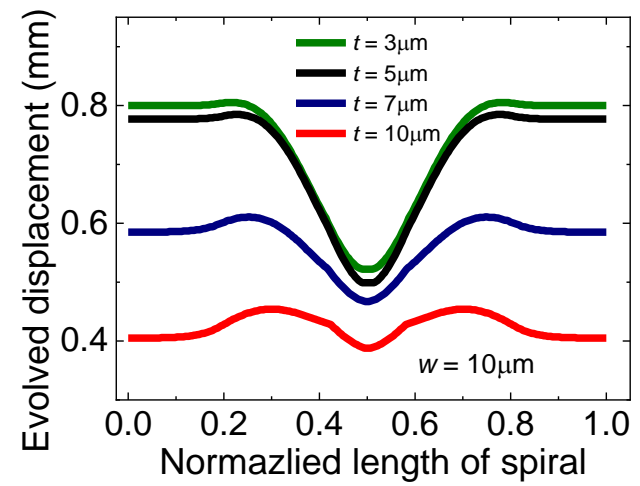

(c)

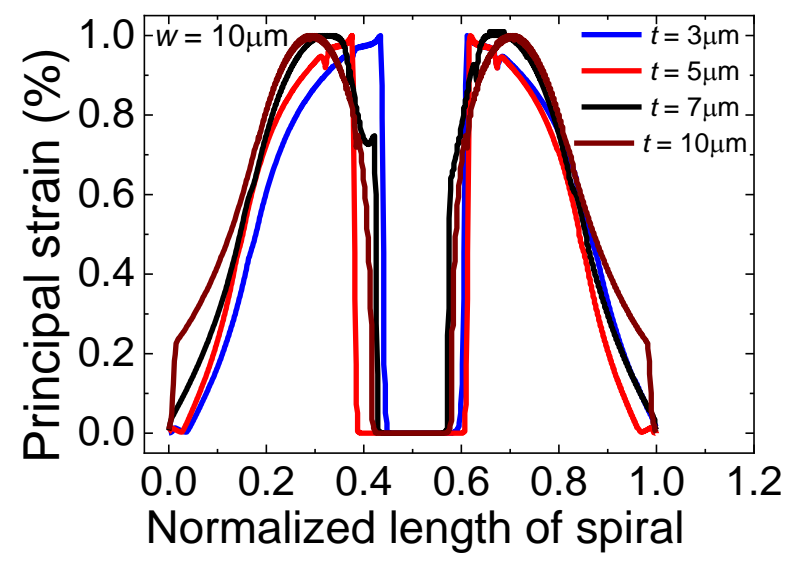

(d) Strain

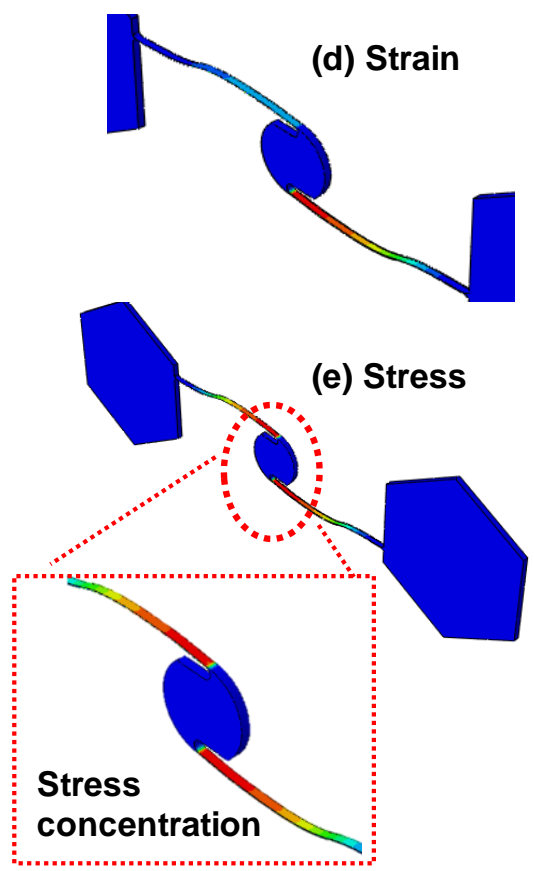

Figure S6. At fracture conditions, mechanical response along the arm of the spiral. (a) Stretched state of the spiral of $t$ of $5 \mu \mathrm{m}$ showing the contours of deformations, whereas (b) shows the evolved displacement along the normalized length of the spiral as a function of $t$. Results reveal that spiral with high $t$ (i.e., $t$ of $10 \mu \mathrm{m}$ ) stretches to less extent than thin, i.e., $t$ of $3 \mu \mathrm{m}$. (c) For various values of $t$, we map the principal strain (\%). (d, e) 3D strain and stress contours are showing the stress concentration areas. Experiments proved that spiral breaks exactly at these stress concentration regions. 


\section{WILEY-VCH}

7- Out-of-plane response horseshoe and spiral, i.e., with $t=3 \mu \mathrm{m}$ and $w=10 \mu \mathrm{m}$

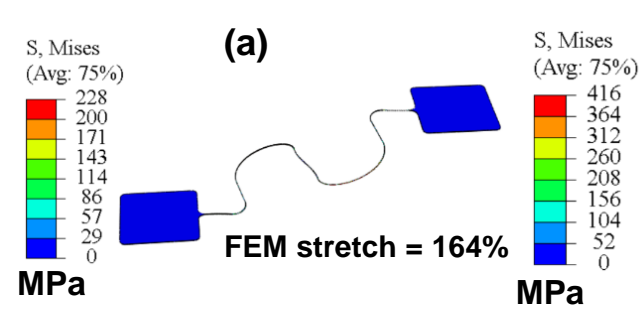

(d)

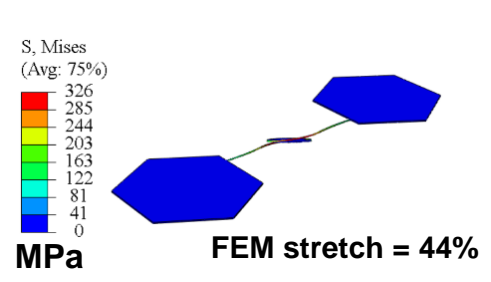

(b)

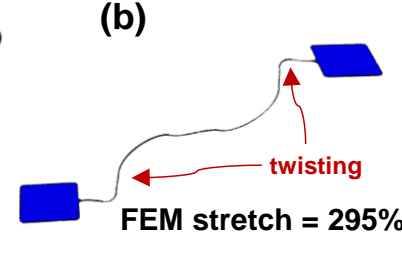

(e)

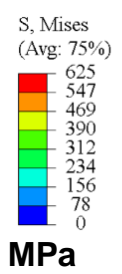

S, Mises

(Avg: $75 \%$ )

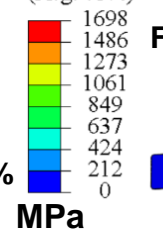

$\mathrm{MPa}$

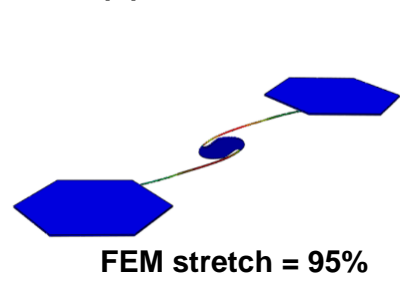

(c)

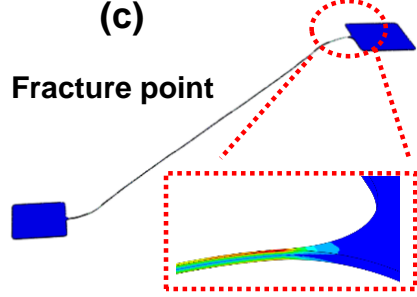

(f)

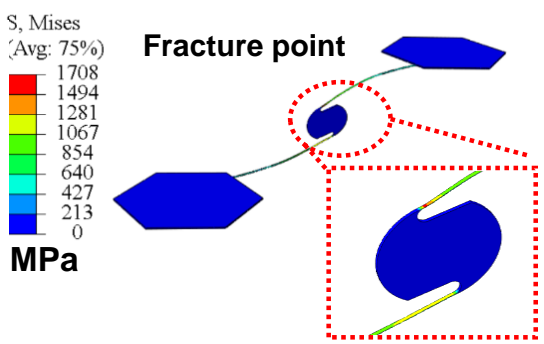

Figure S7. Stress contours for out-of-plane stretching for $\boldsymbol{t}=\mathbf{3} \boldsymbol{\mu \mathrm { m }}, \boldsymbol{w}=\mathbf{1 0} \boldsymbol{\mu \mathrm { m }}$. Numerical calculations show stretching profiles at (a-c) 164\%, 255\%, and fracture conditions for horseshoe, and (d-f) 44\%, 95\%, and fracture conditions for the spiral., respectively. The results show the additional twisting for horseshoe interconnect that leads to low stretchability for thicker interconnect (i.e., for $t=25 \mu \mathrm{m}$, from $184 \%$ for in-plane to $144 \%$ for out-of-plane case). However, the spiral's inner-circle retains the systematic unwrapping of the interconnect during out-of-plane stretching, representing nearly identical stretchability to in-plane stretchability. 


\section{WILEY-VCH}

8- Mechanical response of thicker horseshoe, i.e., with $t=25 \mu \mathrm{m}$

(a)

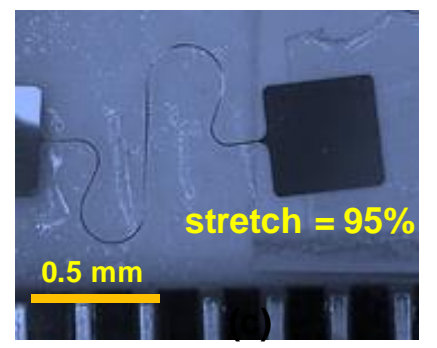

(d)

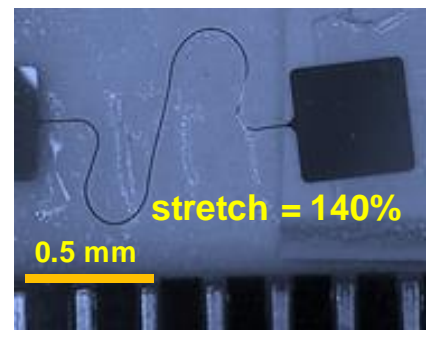

(g) Fractured

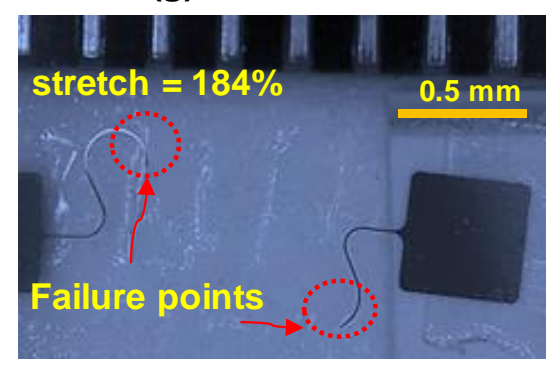

(b)

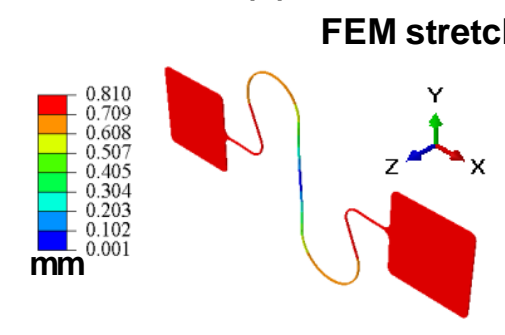

(e)

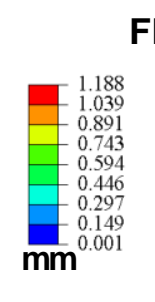

FEM stretch $=140 \%$
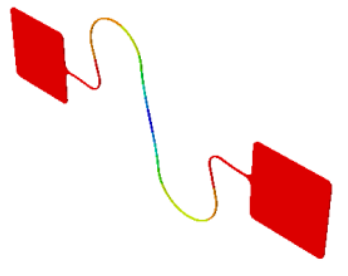

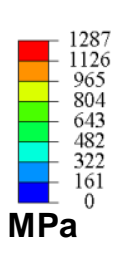

(c)

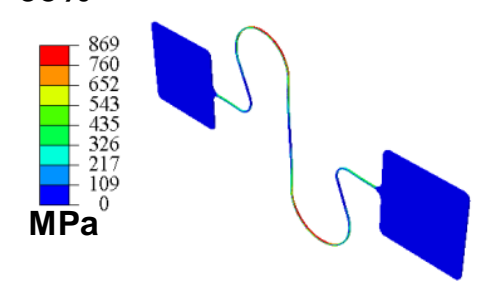

(f)

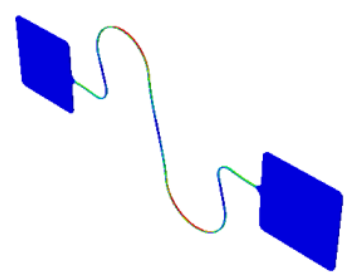

(h) FEM fracture-conditions
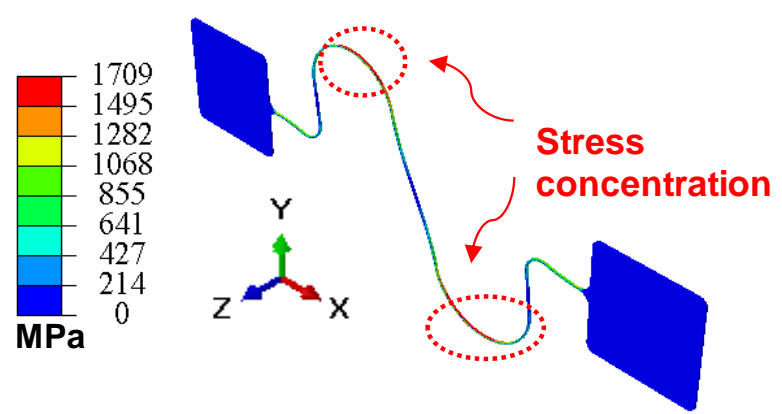

Figure S8. Stretching profiles and fracture conditions of the horseshoe with $t$ of $25 \mu \mathrm{m}$. Experimental stretching and numerical calculations show deformation and stress contours at the applied stretch of (a-c) $95 \%$, and (d-f) 140\%. At fracture-conditions, i.e., a stretch of $184 \%$, $(\mathrm{g}, \mathrm{h})$ broken sample of the fabricated horseshoe and FEM-based prediction of the region that may experience the fracture, i.e., stress concentration. Interestingly, areas shown as red circles validate the claim by FEM. 


\section{WILEY-VCH}

9- Mechanical response of thicker spiral, i.e., with $t=10 \mu \mathrm{m}$

(a)

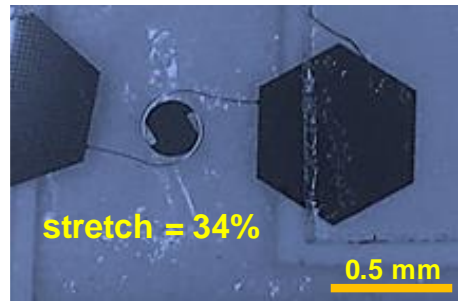

(d)

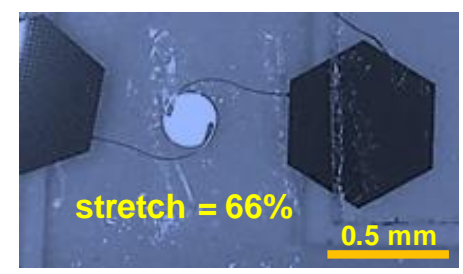

(b)

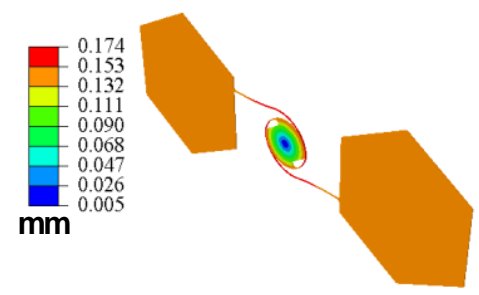

(e)
FEM stretch $=34 \%$

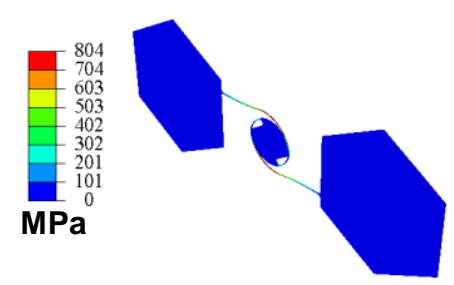

(f)

(c)

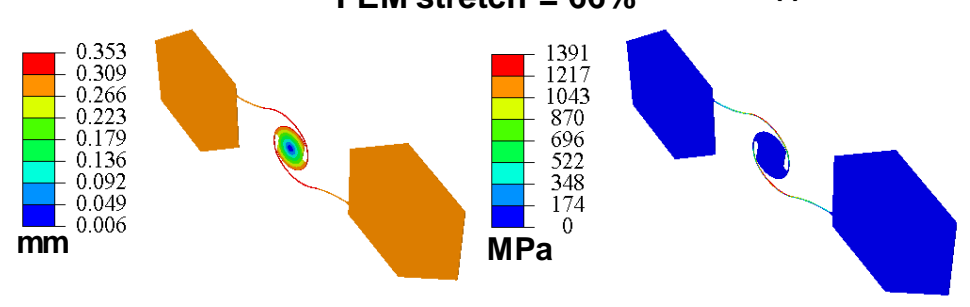

Figure S9. Stretching profiles of the spiral with $\boldsymbol{t}$ of $\mathbf{1 0} \boldsymbol{\mu m}$ before fracture. Experimental stretching profiles, deformation, and von Mises stress based on numerical calculations at the applied stretch of (a-c) 34\% and (d-f) $66 \%$. The spiral was broken in the same areas as predicted by FEM stress concentration (shown in the supplementary movie). 


\section{WILEY-VCH}

10- Normalized stress along the arm of interconnects for various materials

(a)

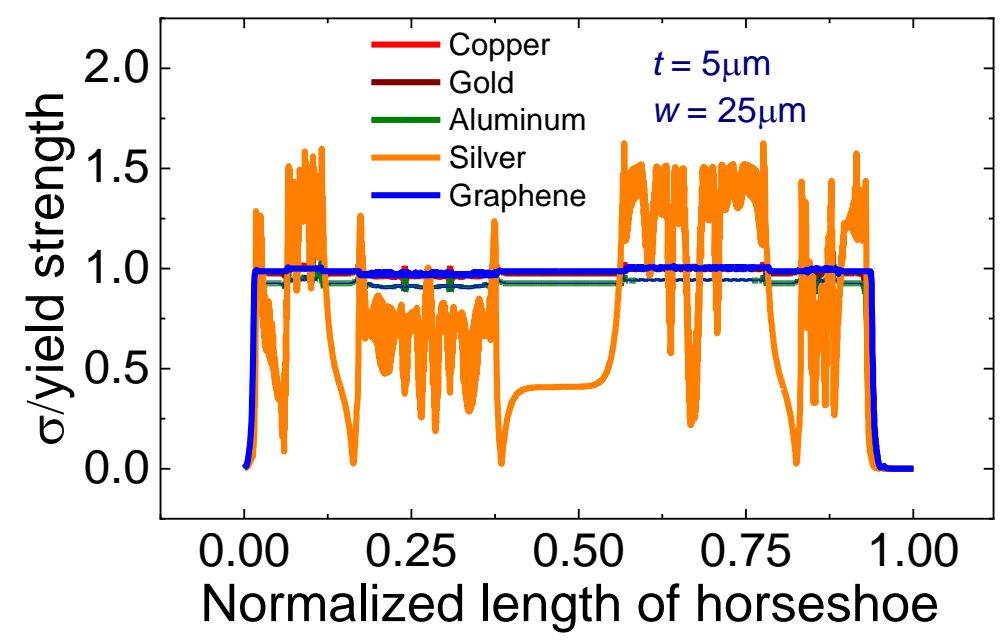

(b)

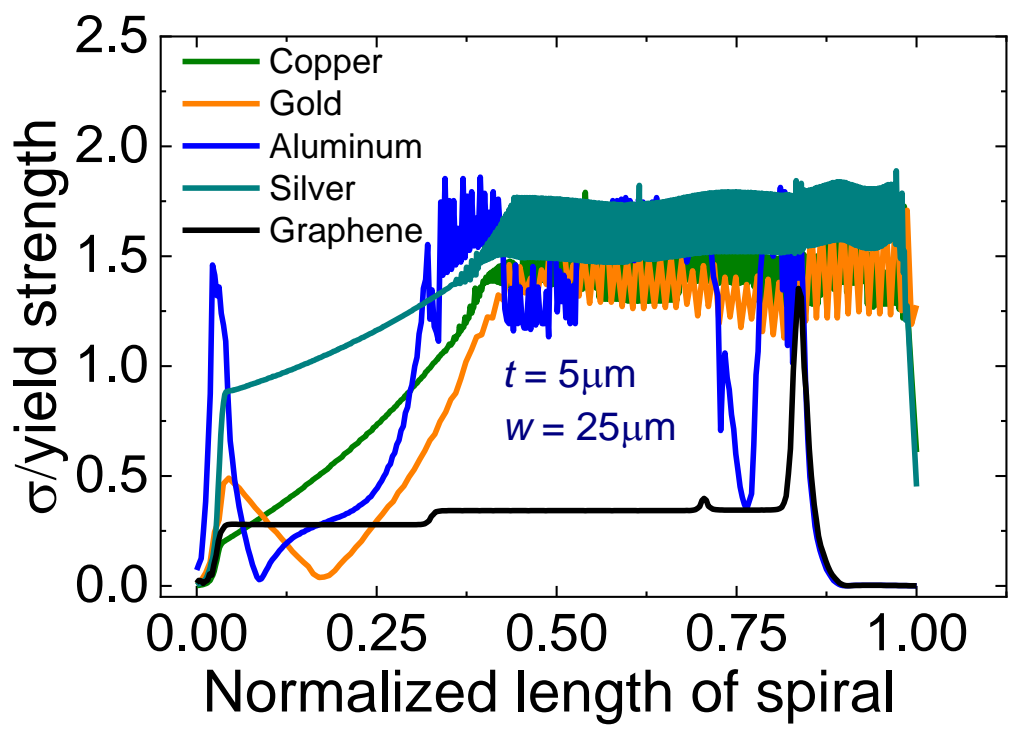

Figure S10. Mechanics along the interconnect arm for various materials. (a, b) Normalized stress along the normalized length of the horseshoe and spiral arm for different materials showing the stress fluctuations or instabilities for the case of silver. The reason for the poor performance of silver is attributed to the unstable stress states during its stretching. 


\section{WILEY-VCH}

Table S1. Intrinsic material properties considered in FEM analysis for commonly used materials for stretchable electronics.

\begin{tabular}{|c|c|c|c|c|}
\hline Material & $\begin{array}{c}\text { Elastic modulus } \\
\text { (GPa) }\end{array}$ & Poisson ratio & $\begin{array}{c}\text { Yield } \\
\text { strength } \\
\text { (MPa) }\end{array}$ & $\begin{array}{c}\text { Fracture strain } \\
\text { (\%) }\end{array}$ \\
\hline Copper & 120 & 0.34 & 220 & 5 \\
\hline Gold & 70 & 0.44 & 210 & 5 \\
\hline Silver & 83 & 0.37 & 54 & 2 \\
\hline Graphene & 1000 & 0.2 & 150 & 22 \\
\hline Aluminum & 70 & 0.35 & 150 & 20 \\
\hline Silicon & 170 & 0.22 & - & 1 \\
\hline
\end{tabular}

